\title{
Synthesis of Orthogonally Protected Labionin
}

\author{
Eliana Lo Presti, § Alessandro Volonterio ${ }^{\dagger} \S$, Monica Sani ${ }^{\star}, \S$
}

\$National Research Council, Institute of Chemical Sciences and Technologies "Giulio Natta" (SCITEC), Via Mario Bianco 9, 20131 Milan, Italy.

†Department of Chemistry, Materials, and Chemical Engineering "Giulio Natta", Politecnico di Milano, via Mancinelli 7, 20141 Milano, Italy.

monica.sani@polimi.it

\section{Table of contents}

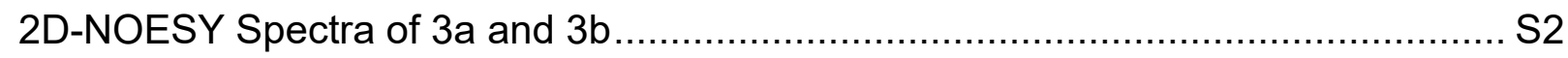

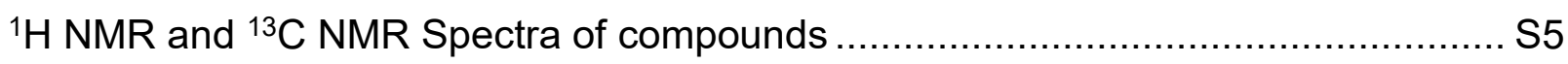

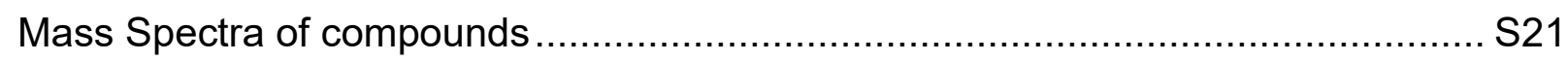




\section{D-NOESY Spectra of $3 a$ and $3 b$}

Absolute stereochemistry for compound $\mathbf{3} \mathbf{a}$ and $\mathbf{3 b}$ was assigned by 2D-NOESY-NMR spectroscopy. In both derivatives, the relative position of $\mathrm{Ha}$ and $\mathrm{Hb}$ with respect to the lactam ring plan was identified by measuring their coupling constants with $\mathrm{Hc}$. In derivative 3a, Ha exhibits a $\mathrm{J}_{a b}=14.1 \mathrm{~Hz}$ and $\mathrm{a} \mathrm{Jac}_{\mathrm{ac}}=9.4 \mathrm{~Hz}$, while $\mathrm{Hb}$ shows a significantly lower $\mathrm{Jbc}_{\mathrm{bc}}$ $=4.9 \mathrm{~Hz}$, suggesting that $\mathrm{Hb}$ is oriented on the opposite face of the pyroglutamic plan with respect to $\mathrm{Hc}$. The presence of a NOE effect between $\mathrm{Hb}$ and $\mathrm{Hd}$ indicates that they are positioned on the same face of the molecular plan, permitting the attribution of the absolute stereochemistry of the newly generated quaternary carbon center as $R$ (Figure $\mathrm{S} 1$ and S2). The same approach was used for the derivative $3 \mathbf{b}$. Hb shows a Jba $=13.9 \mathrm{~Hz}$ and a $\mathrm{J}_{\mathrm{bc}}=2.3 \mathrm{~Hz}$, while Ha showed a $\mathrm{J}_{\mathrm{ab}}$ of $13.9 \mathrm{~Hz}$ and a $\mathrm{J}_{\mathrm{ac}}=9.7 \mathrm{~Hz}$, suggesting that $\mathrm{Ha}$ is oriented on the same face of the pyroglutamic plan with respect to Hc. The presence of a NOE effect between $\mathrm{Ha}$ and $\mathrm{Hd}$ indicates that they are positioned on the same face of the molecular plan, permitting the attribution of the absolute stereochemistry of the newly generated quaternary carbon center as $S$ (Figure S1 and S3).

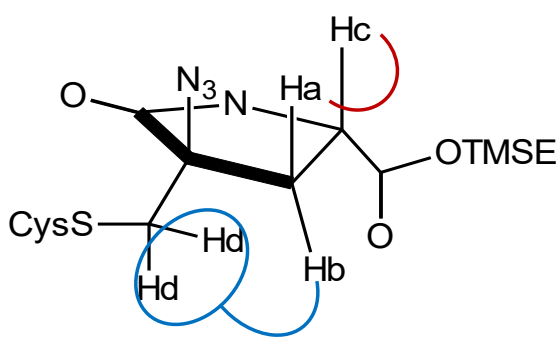

3a

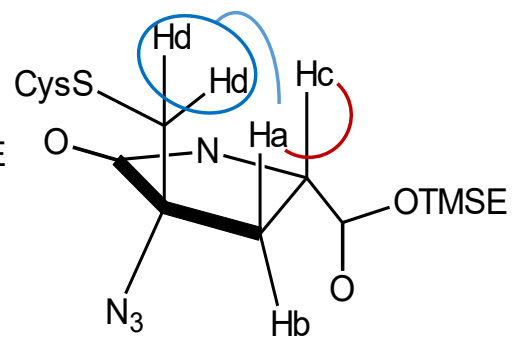

$3 b$

Figure $\mathbf{S 1}$ : Skew representation of $\mathbf{3} \mathbf{a}$ and $\mathbf{3 b}$. 


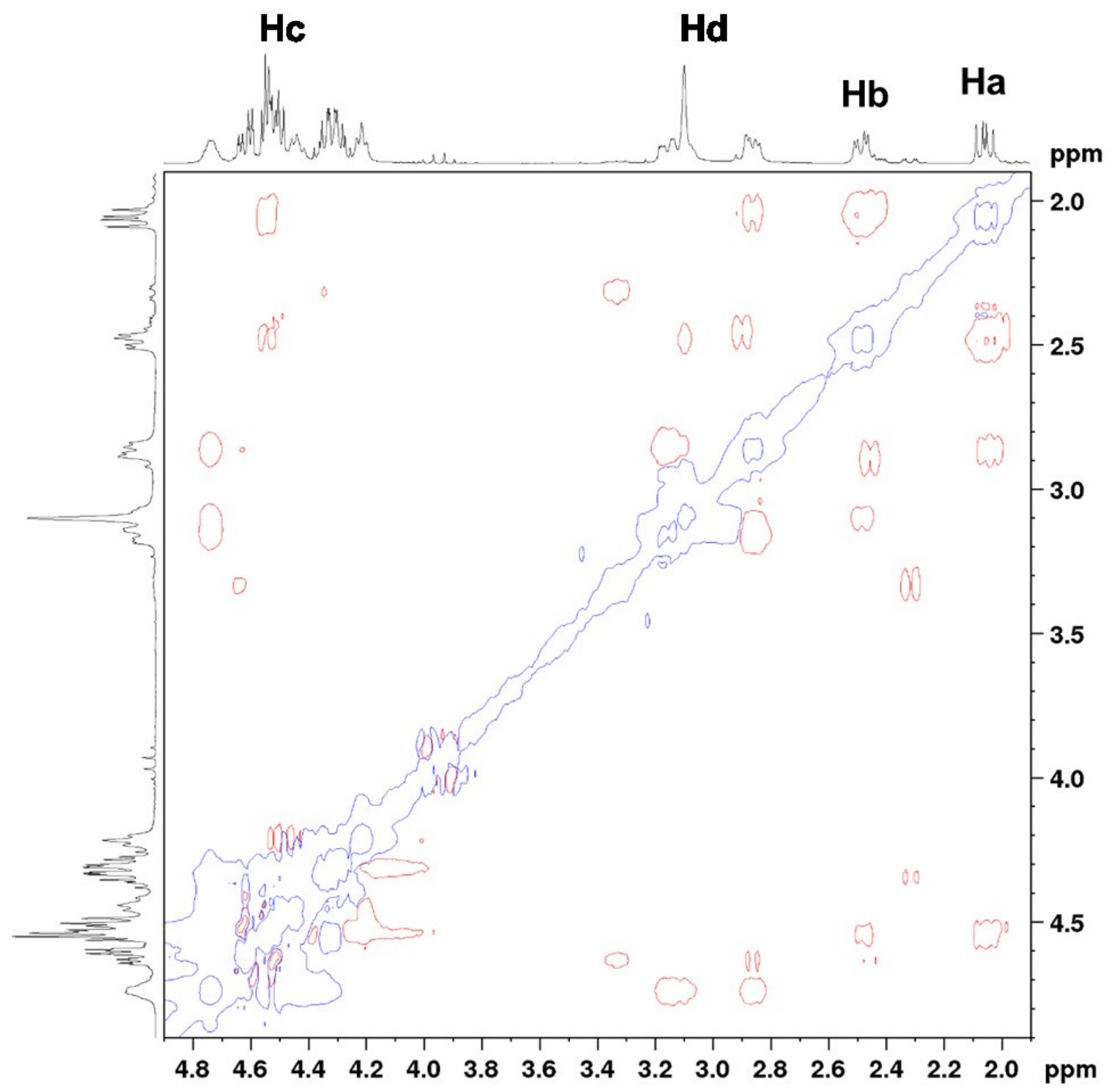

Figure S2: 2D-NOESY-NMR spectrum of compound 3a, benzene $\mathrm{d}^{6}$, mixing time $800 \mathrm{~ms}$. 


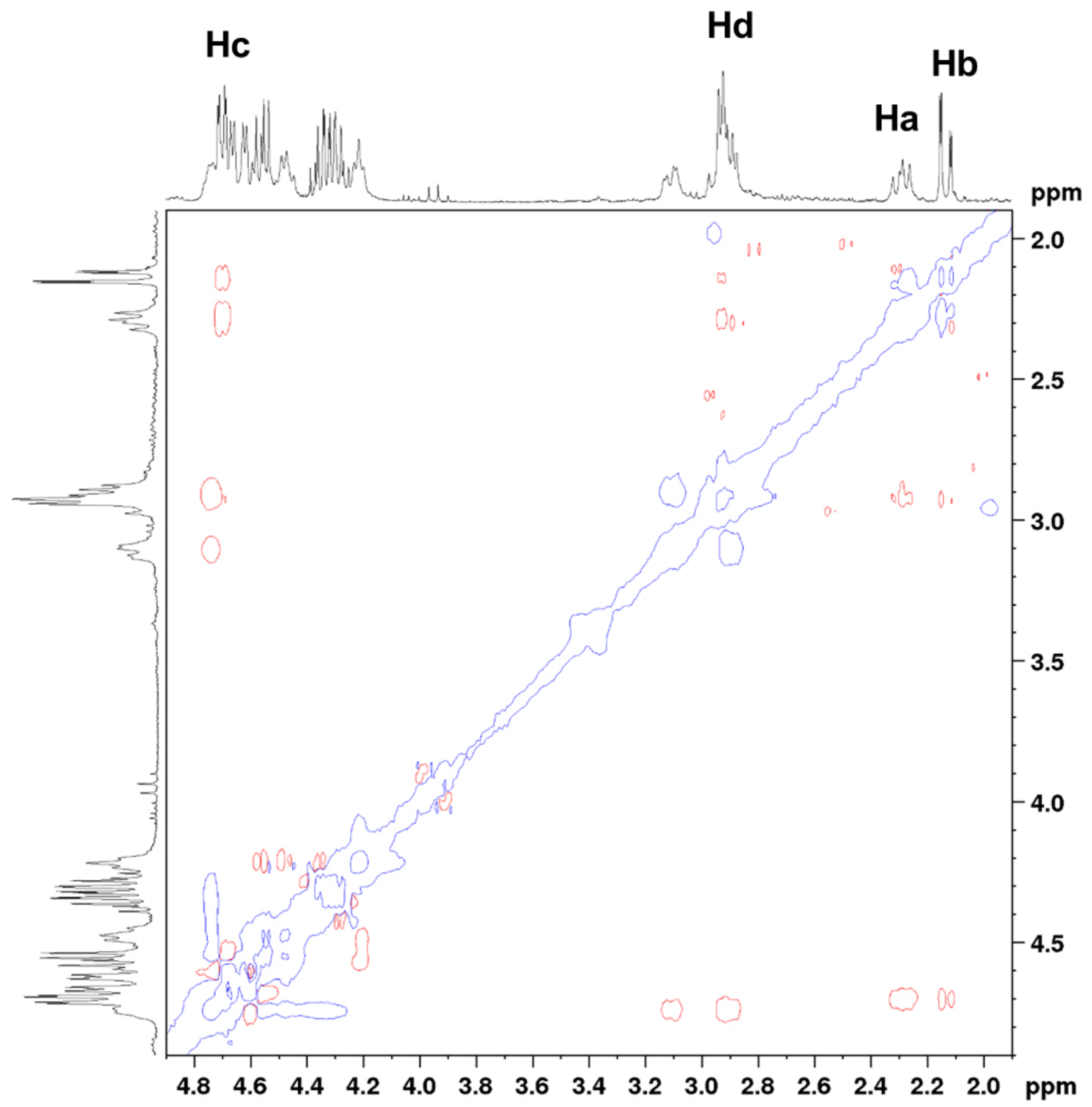

Figure S3: 2D-NOESY-NMR spectrum of compound $\mathbf{3 b}$, benzene $\mathrm{d}^{6}$, mixing time $800 \mathrm{~ms}$. 


\section{${ }^{1} \mathrm{H}$ NMR and ${ }^{13} \mathrm{C}$ NMR Spectra of compounds}

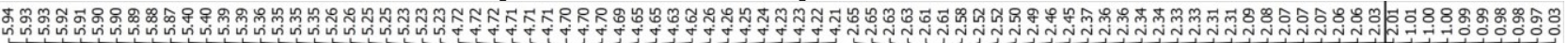

${ }^{1} \mathrm{H} \mathrm{NMR}\left(\mathrm{CDCl}_{3}, 400 \mathrm{MHz}\right)$
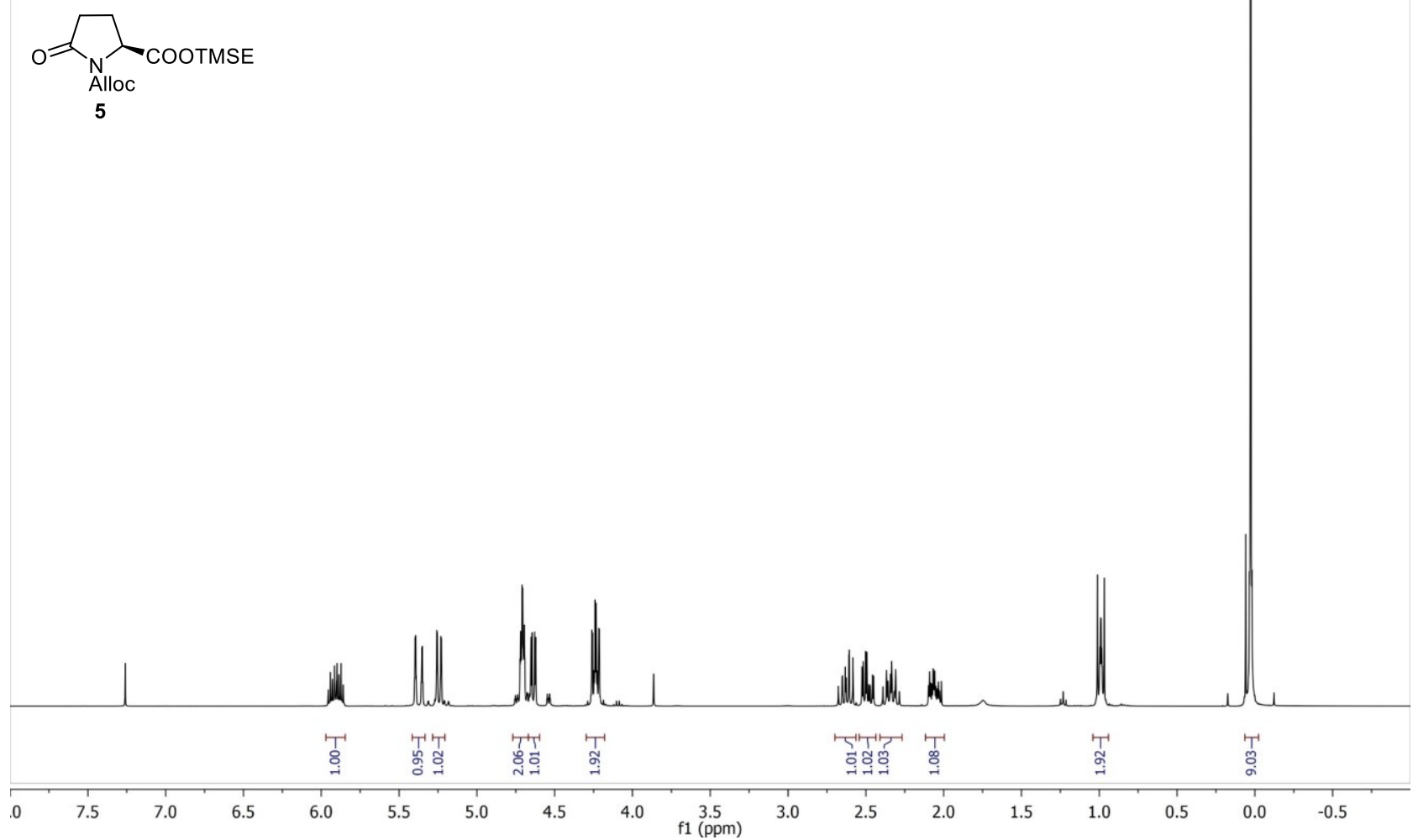

${ }^{13} \mathrm{C}\{1 \mathrm{H}\} \mathrm{NMR}\left(\mathrm{CDCl}_{3}, 101 \mathrm{MHz}\right)$

$\overbrace{\substack{\text { Alloc } \\ \text { Cootmse }}}$

5
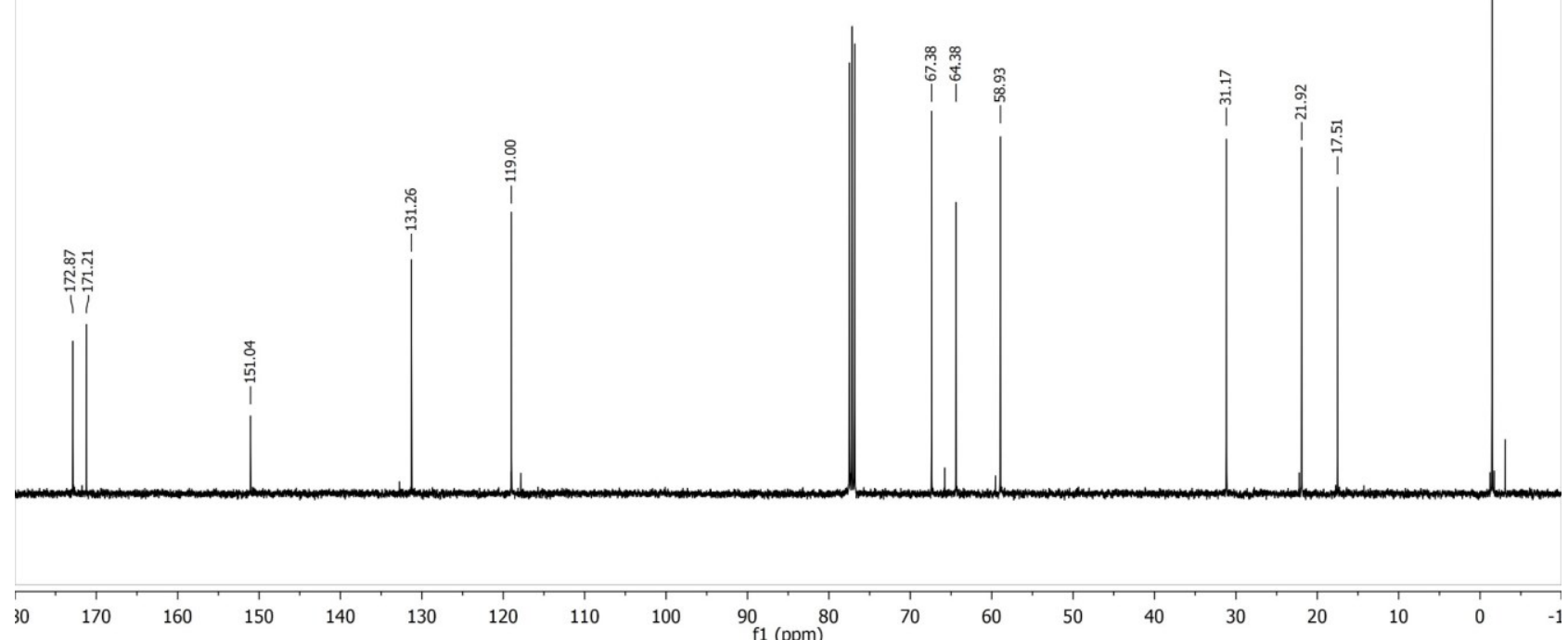
年8

${ }^{1} \mathrm{H}$ NMR $\left(\mathrm{CDCl}_{3}, 400 \mathrm{MHz}\right)$

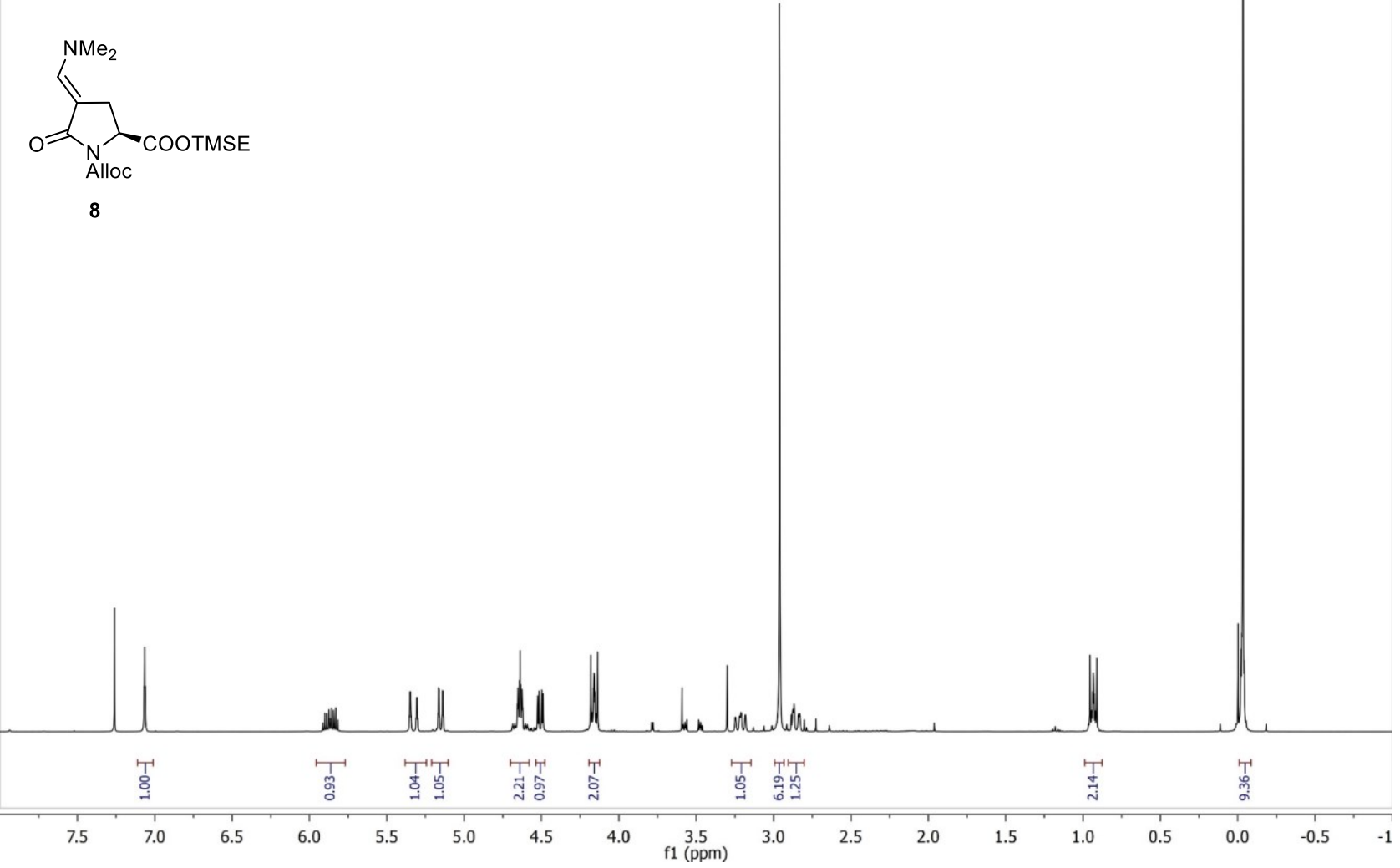

${ }^{13} \mathrm{C}\{1 \mathrm{H}\} \mathrm{NMR}\left(\mathrm{CDCl}_{3}, 101 \mathrm{MHz}\right)$
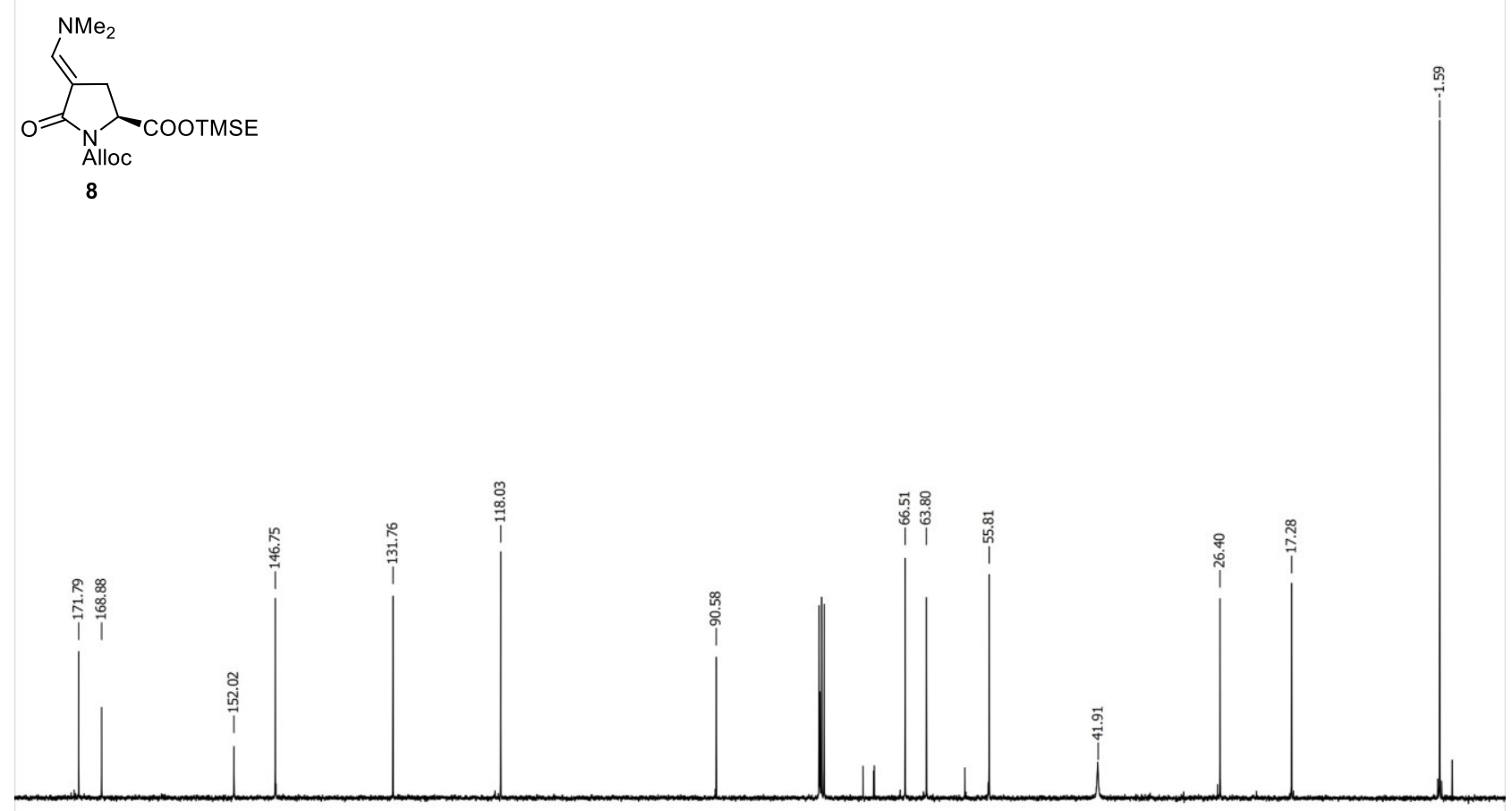

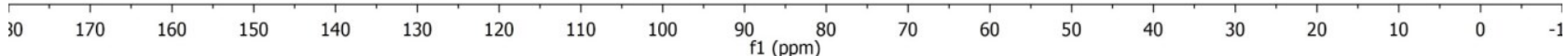




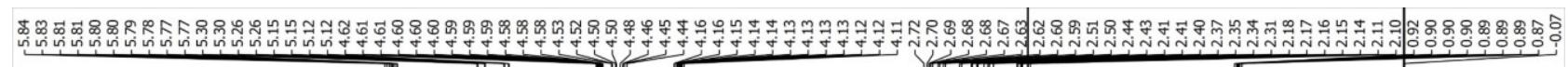

${ }^{1} \mathrm{H} \operatorname{NMR}\left(\mathrm{CDCl}_{3}, 400 \mathrm{MHz}\right)$

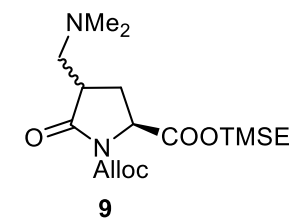

9

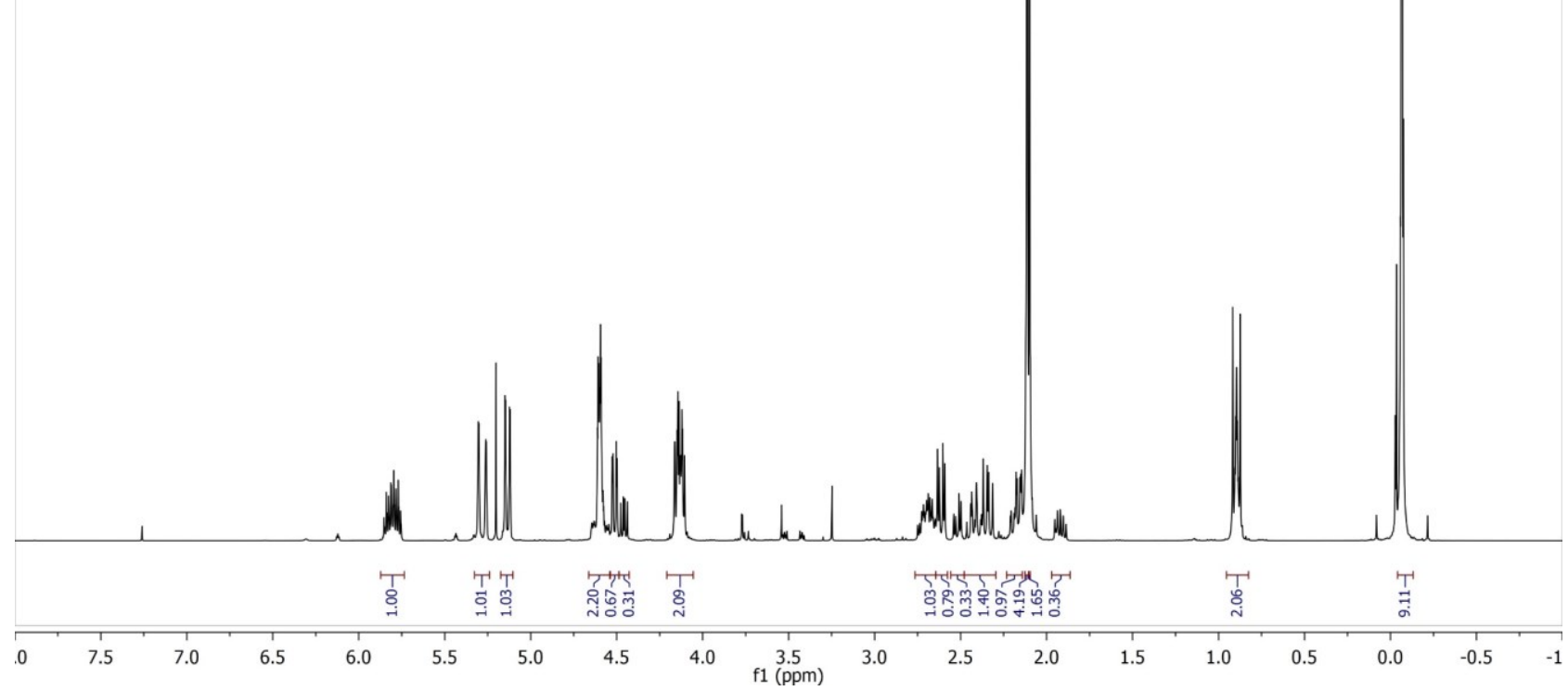

\section{${ }^{13} \mathrm{C}\{1 \mathrm{H}\}$ NMR $\left(\mathrm{CDCl}_{3}, 101 \mathrm{MHz}\right)$}
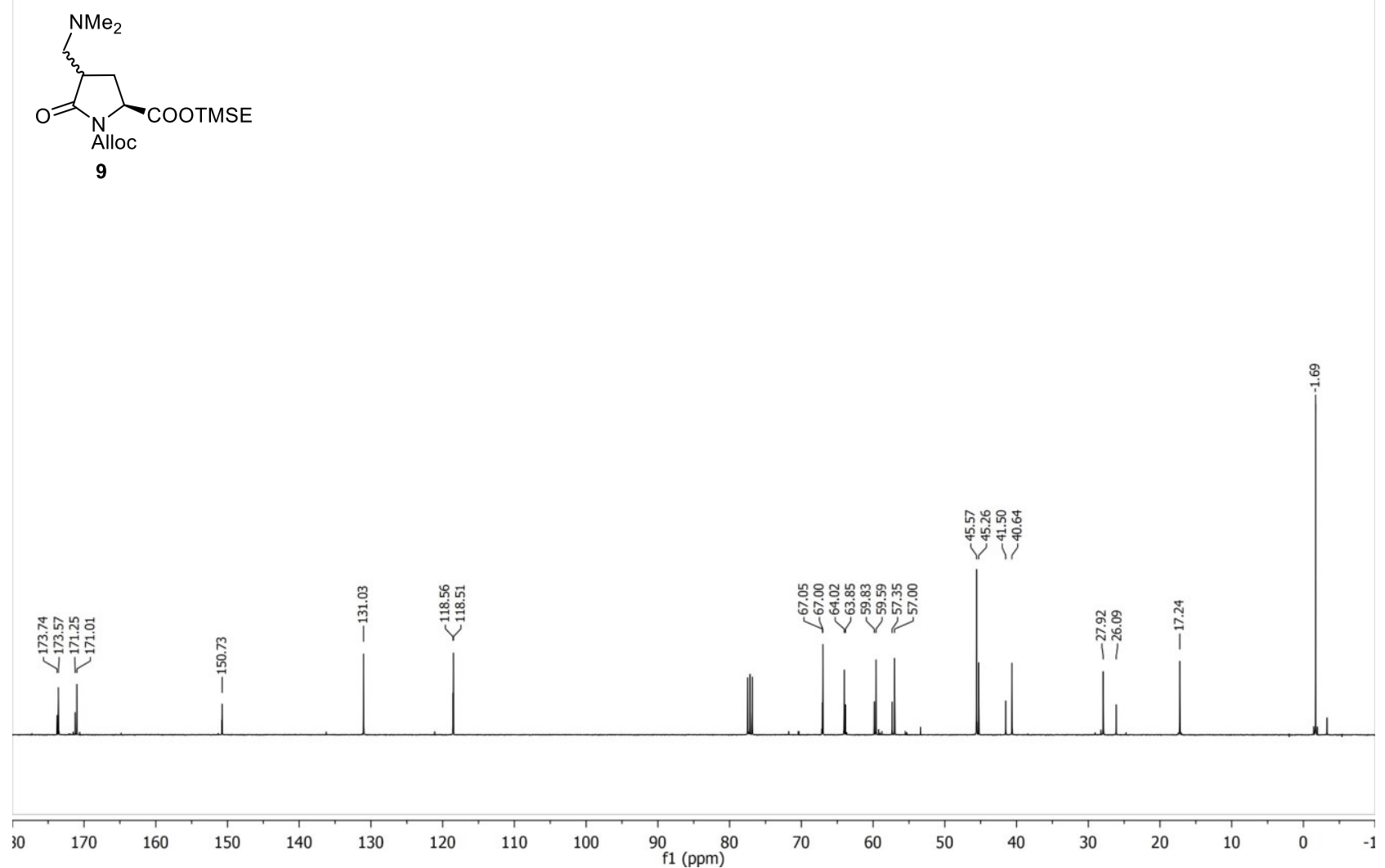
${ }^{1} \mathrm{H}$ NMR $\left(\mathrm{CDCl}_{3}, 400 \mathrm{MHz}\right)$
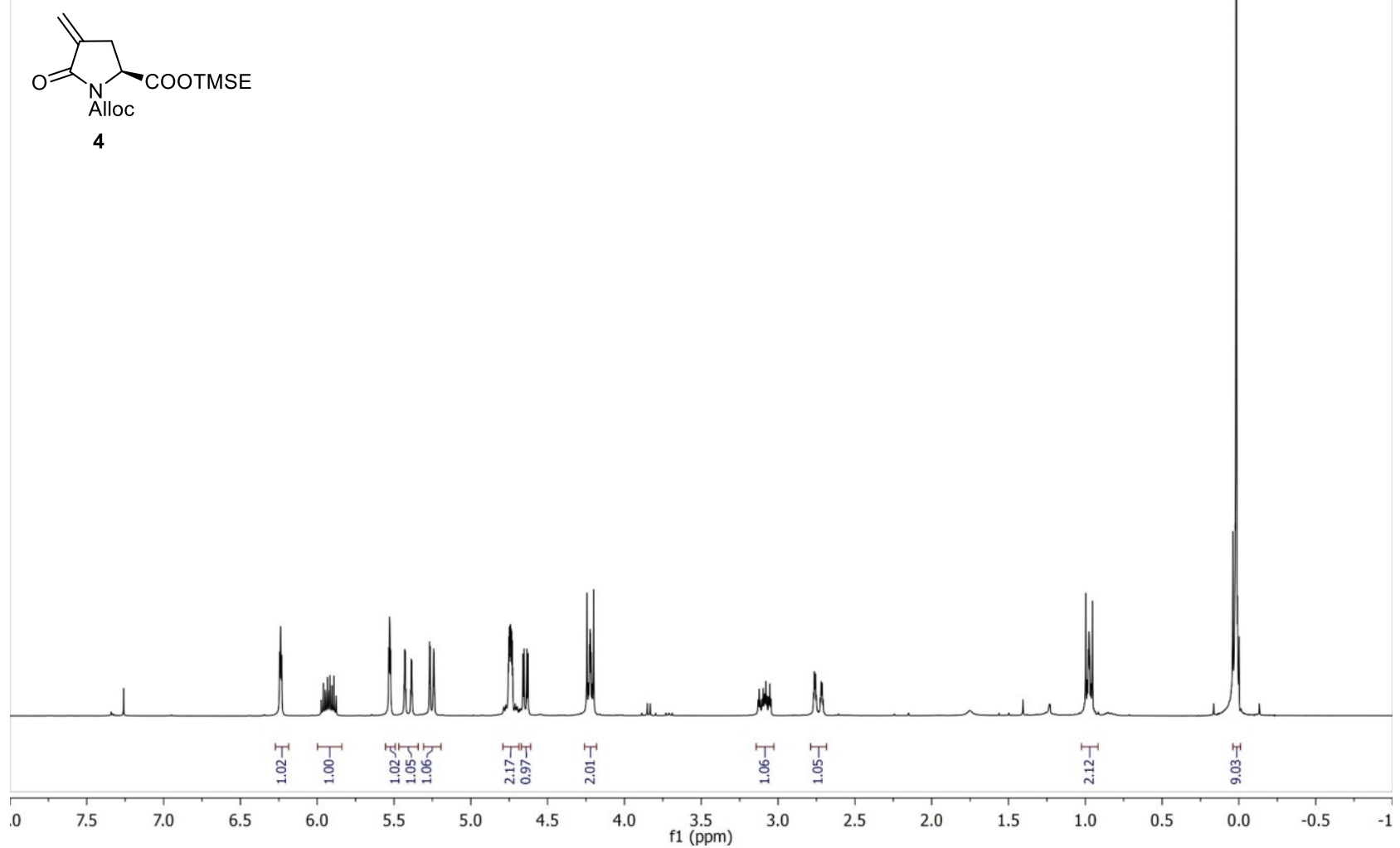

${ }^{13} \mathrm{C}\{1 \mathrm{H}\} \mathrm{NMR}\left(\mathrm{CDCl}_{3}, 101 \mathrm{MHz}\right)$
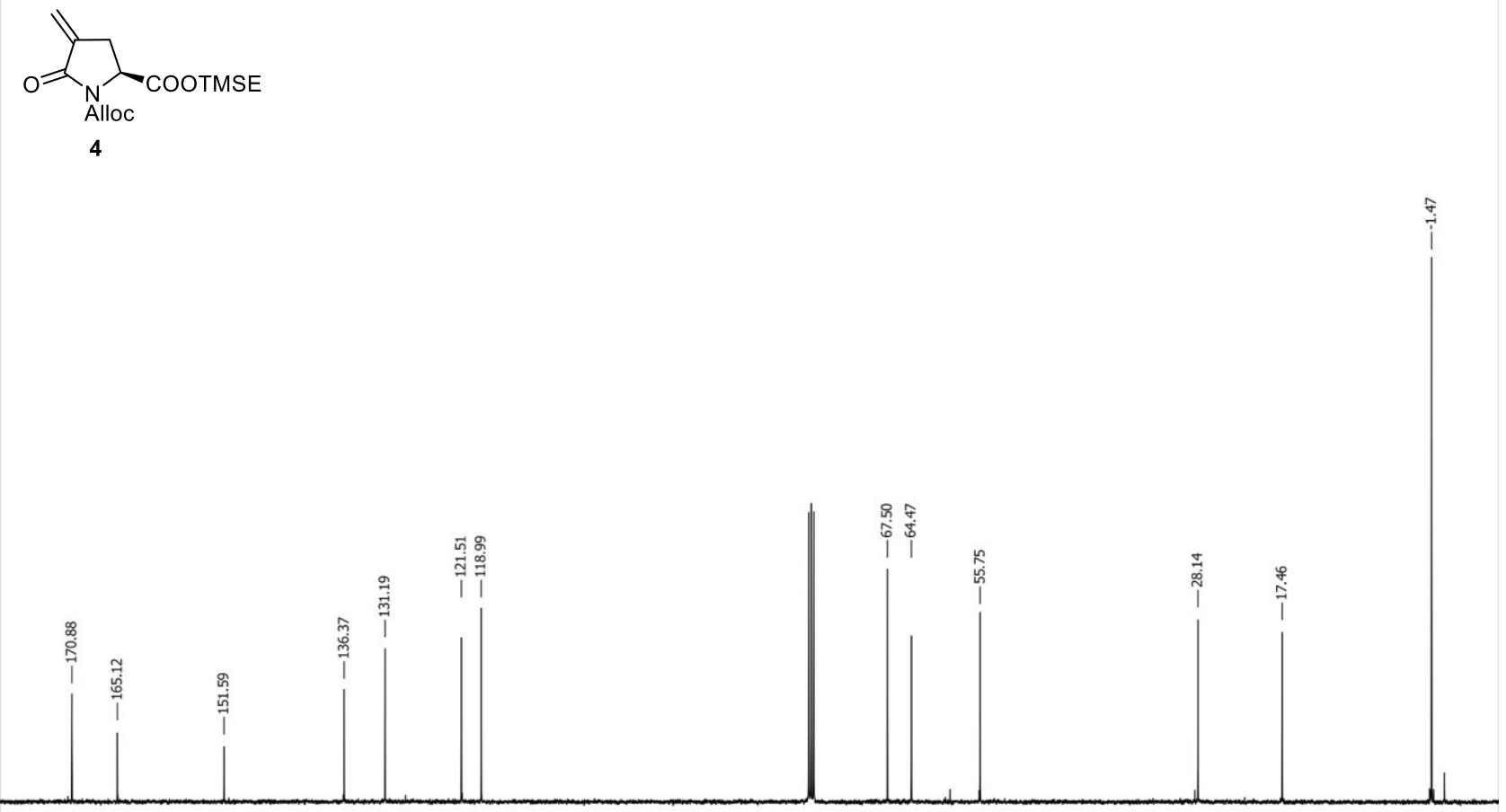

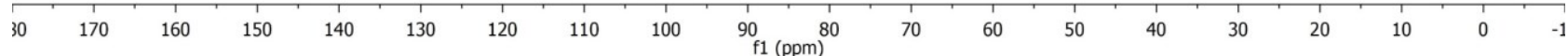


${ }^{1} \mathrm{H} \operatorname{NMR}\left(\mathrm{CDCl}_{3}, 400 \mathrm{MHz}\right)$<smiles>CSC[C@H](NC(F)F)C(=O)OC(C)(C)c1ccccc1</smiles>

11

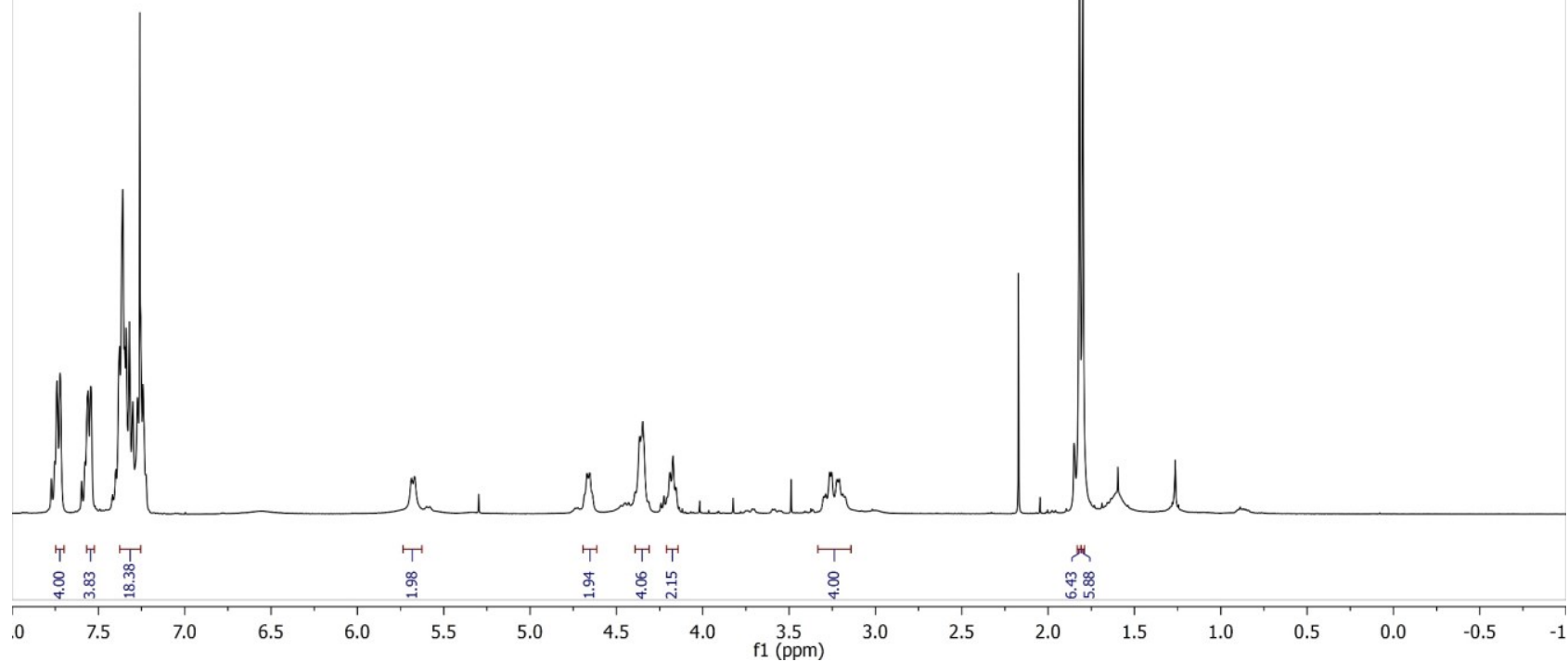

${ }^{13} \mathrm{C}\{1 \mathrm{H}\}$ NMR $\left(\mathrm{CDCl}_{3}, 101 \mathrm{MHz}\right)$

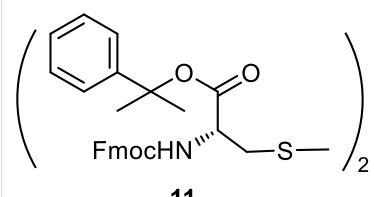

11

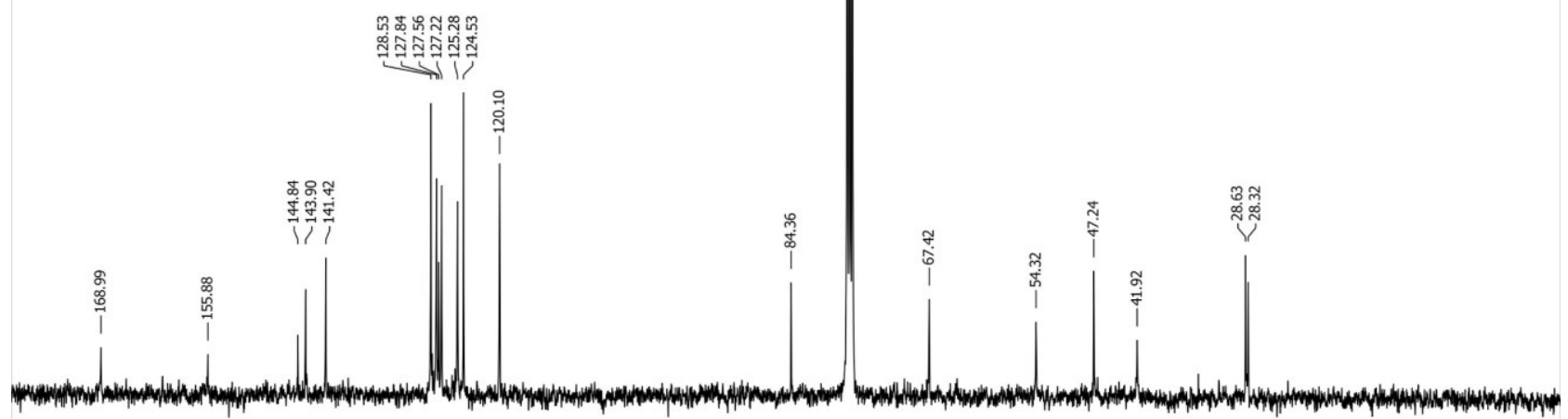

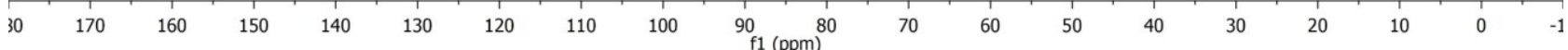


${ }^{1} \mathrm{H} \operatorname{NMR}\left(\mathrm{CDCl}_{3}, 400 \mathrm{MHz}\right)$

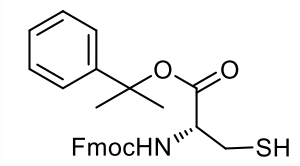

12

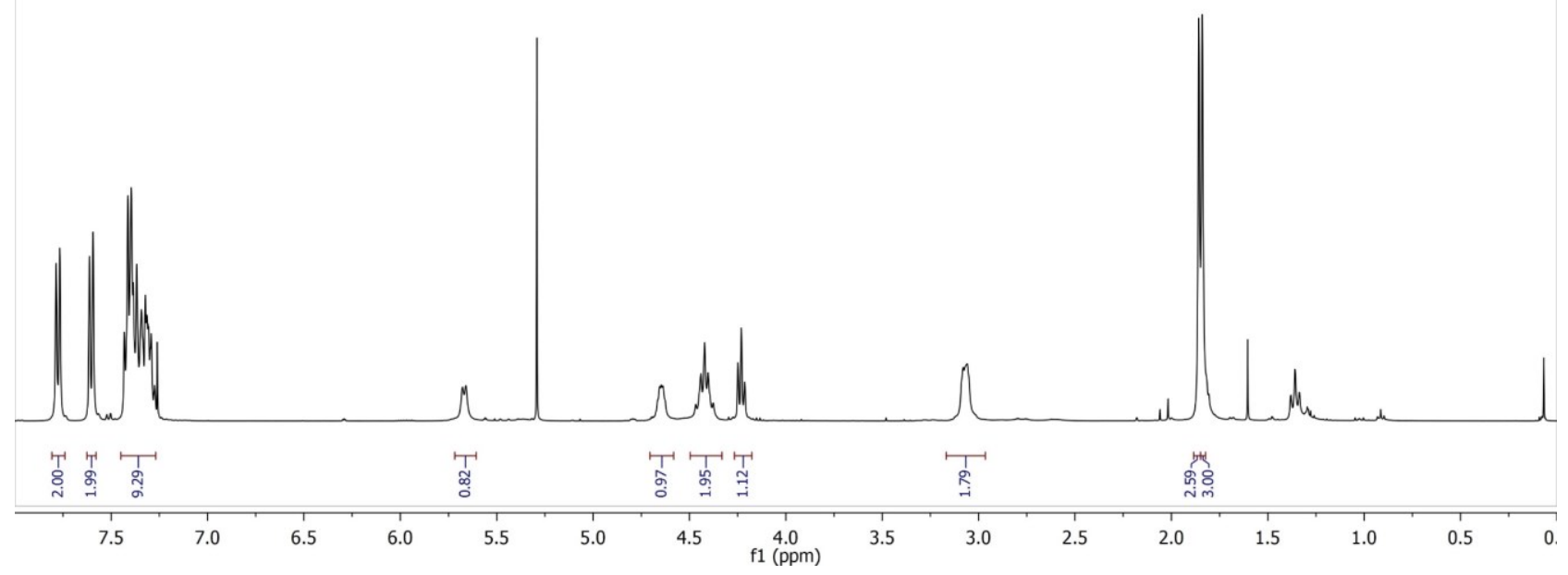

${ }^{13} \mathrm{C}\{1 \mathrm{H}\} \mathrm{NMR}\left(\mathrm{CDCl}_{3}, 101 \mathrm{MHz}\right)$

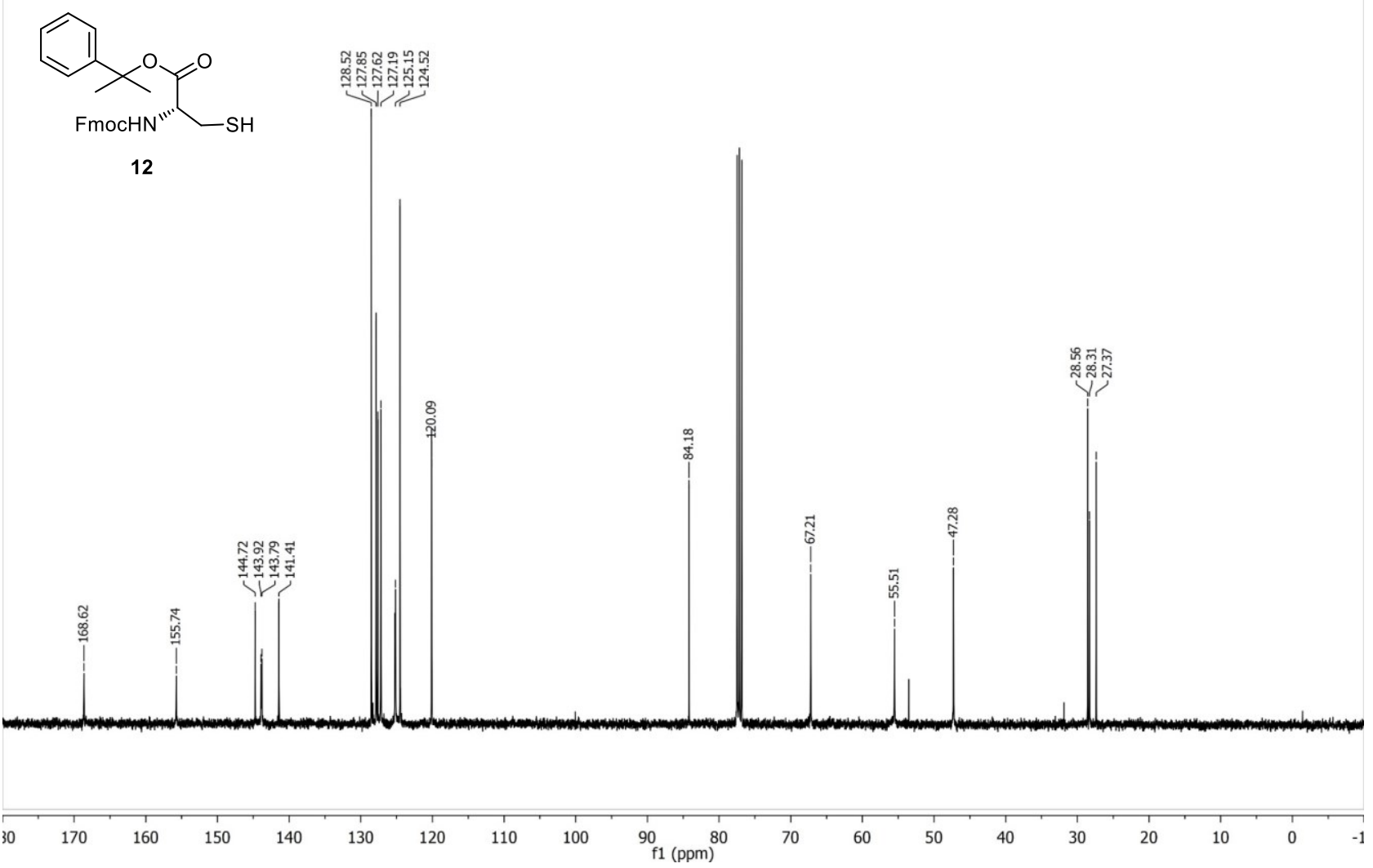




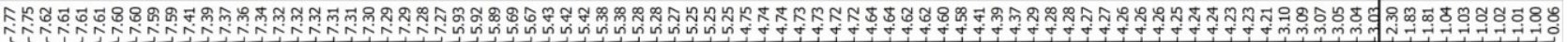

${ }^{1} \mathrm{H}$ NMR $\left(\mathrm{CDCl}_{3}, 400 \mathrm{MHz}\right)$

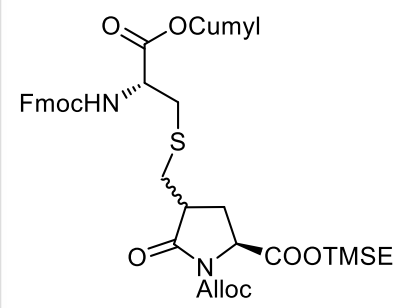

13 a
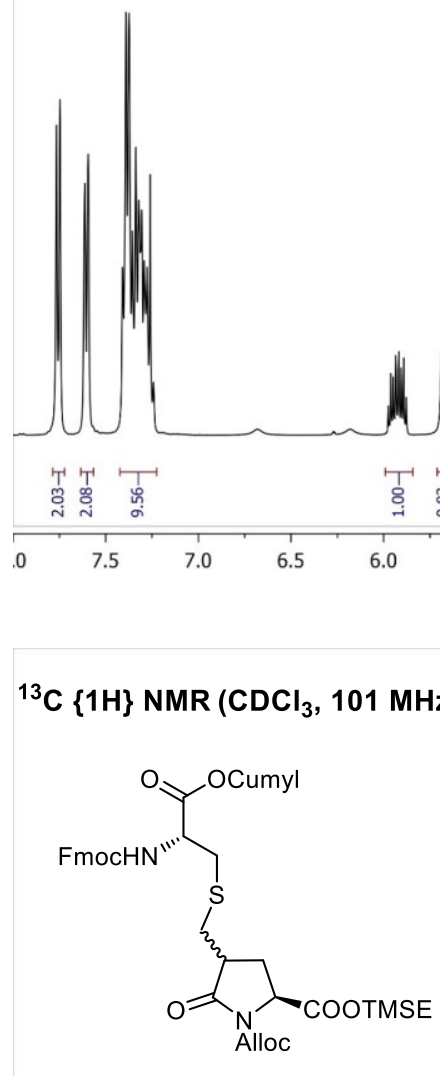

13 a

\section{${ }^{13} \mathrm{C}\{1 \mathrm{H}\} \mathrm{NMR}\left(\mathrm{CDCl}_{3}, 101 \mathrm{MHz}\right)$}
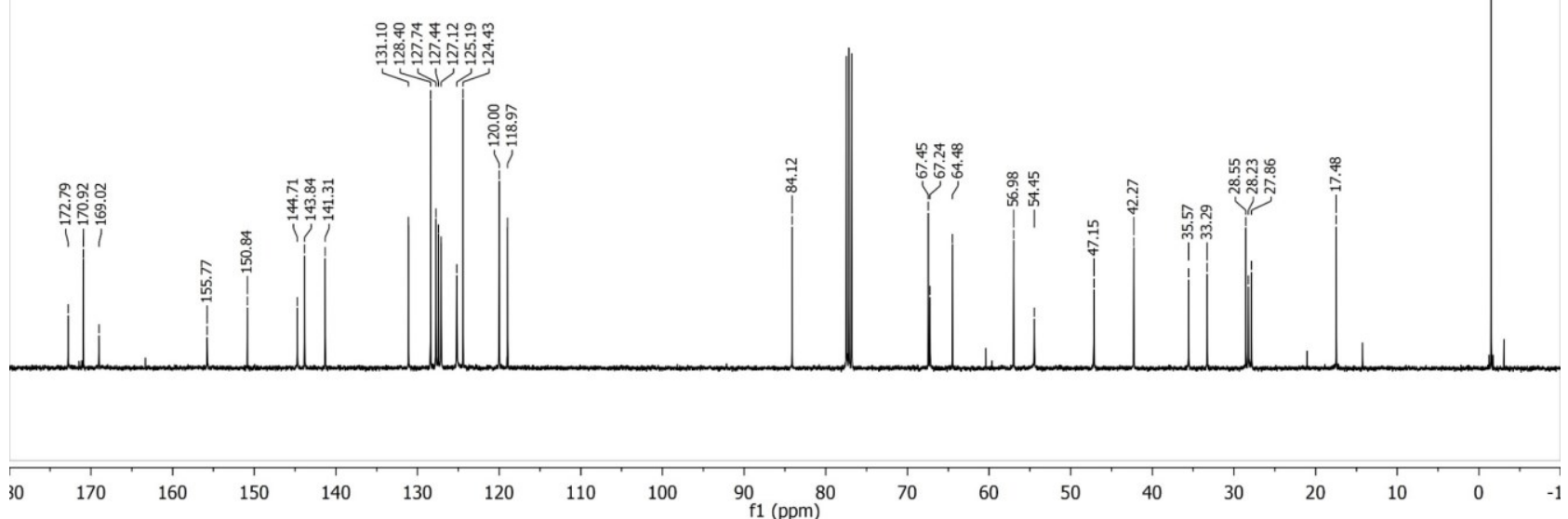
${ }^{1} \mathrm{H}$ NMR $\left(\mathrm{CDCl}_{3}, 400 \mathrm{MHz}\right)$

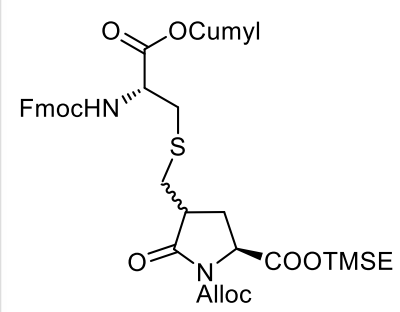

$13 \mathrm{~b}$

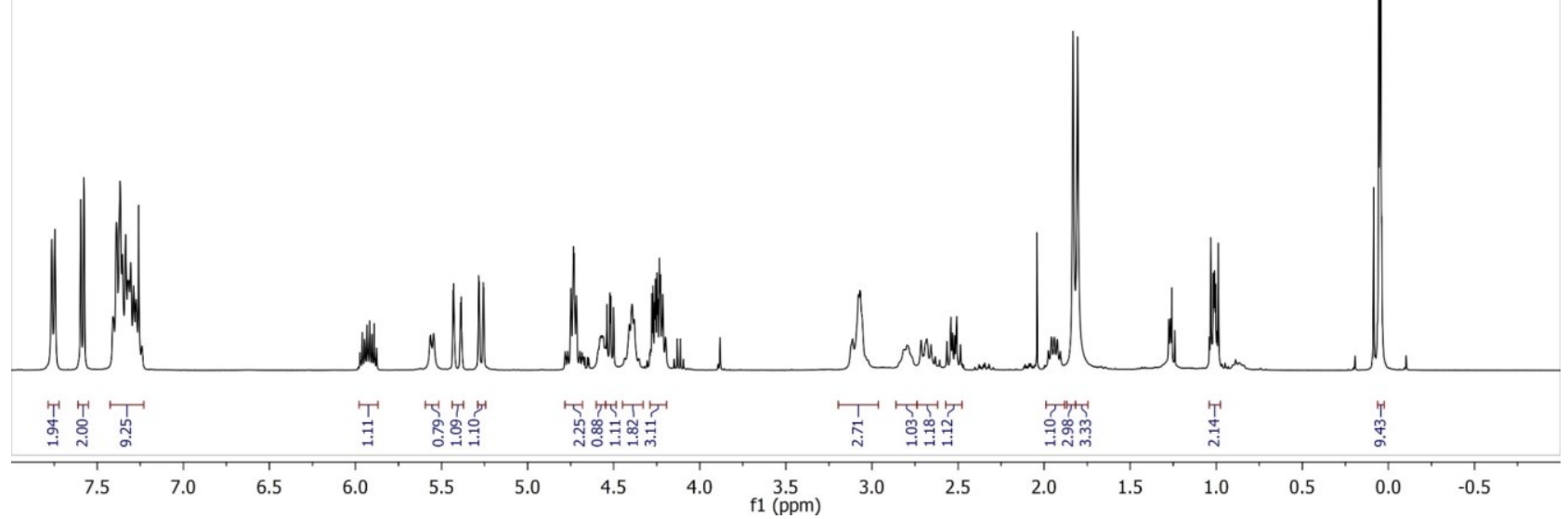

${ }^{13} \mathrm{C}\{1 \mathrm{H}\} \mathrm{NMR}\left(\mathrm{CDCl}_{3}, 101 \mathrm{MHz}\right)$

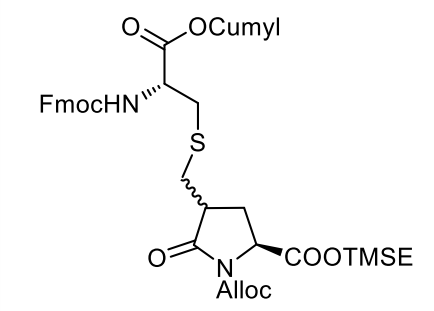

$13 \mathrm{~b}$
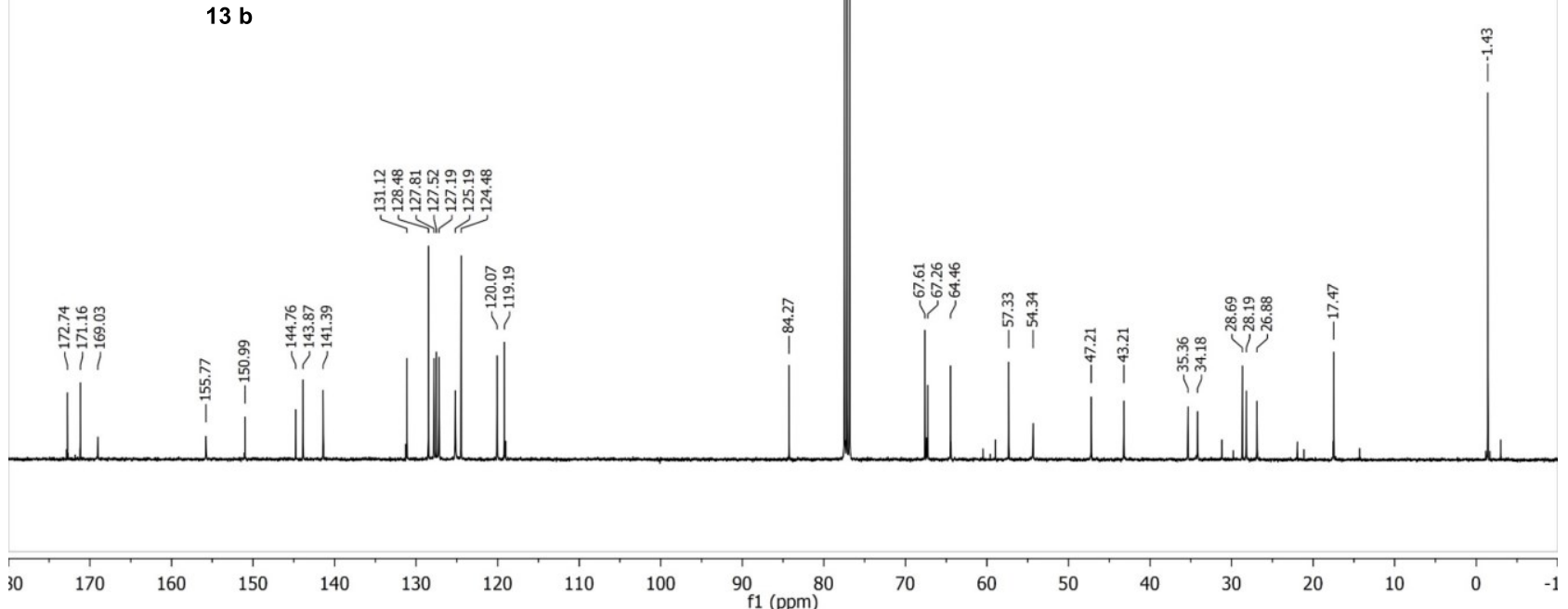


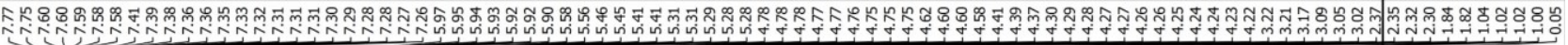

${ }^{1} \mathrm{H}$ NMR $\left(\mathrm{CDCl}_{3}, 400 \mathrm{MHz}\right)$

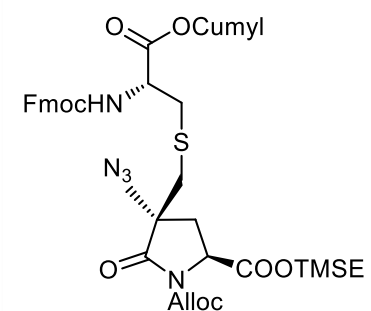

$3 a$

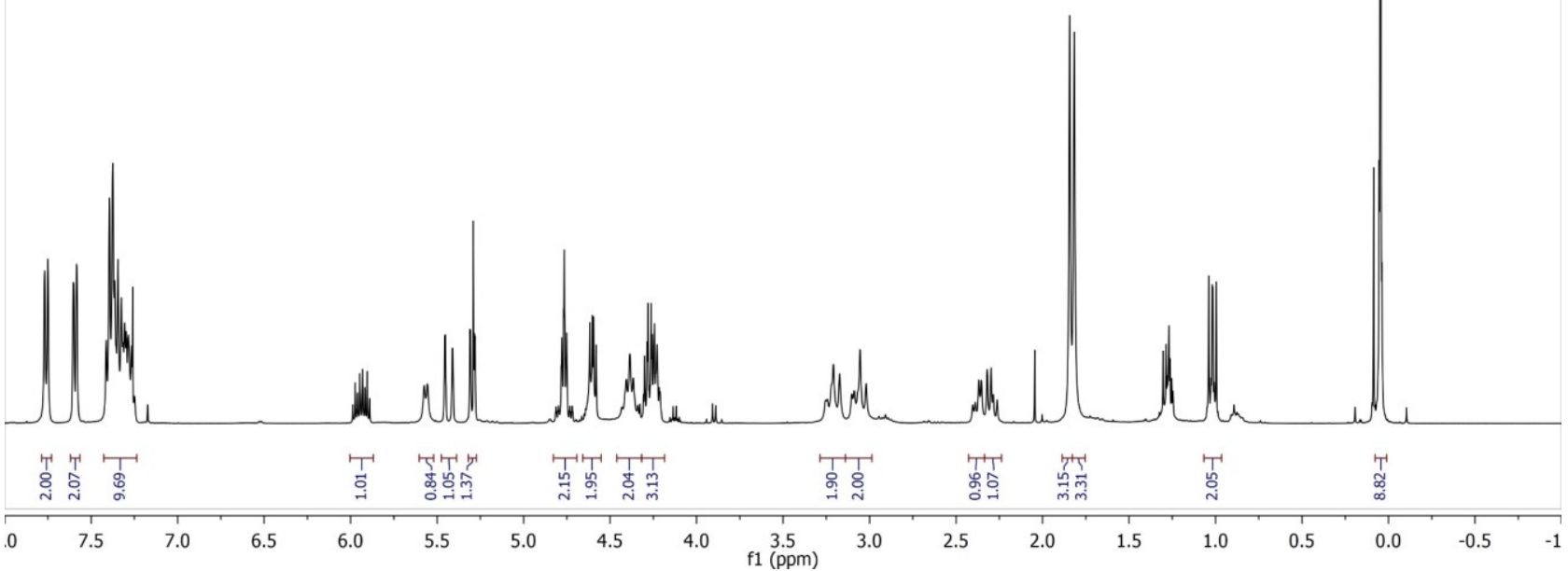

${ }^{13} \mathrm{C}\{1 \mathrm{H}\} \mathrm{NMR}\left(\mathrm{CDCl}_{3}, 101 \mathrm{MHz}\right)$

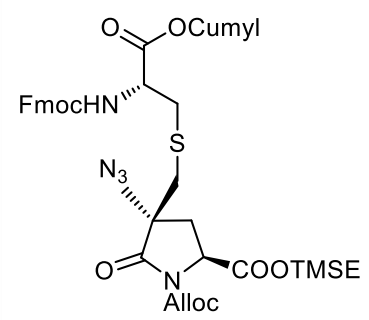

3a

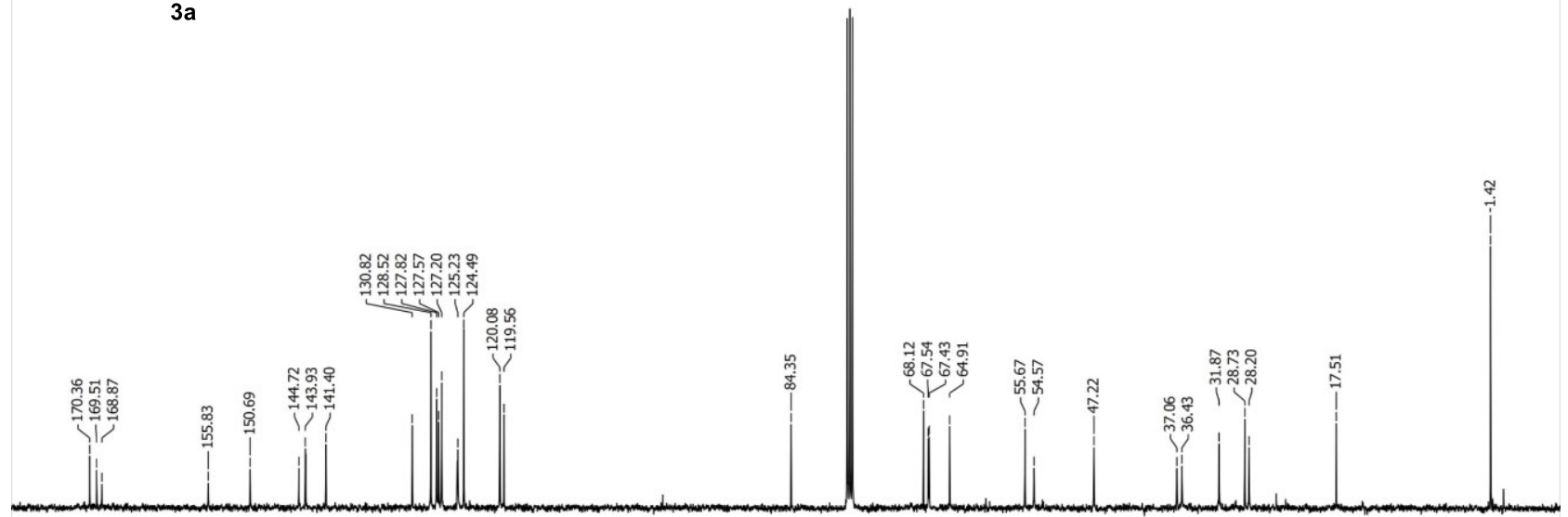

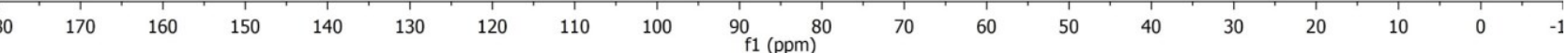


${ }^{1} \mathrm{H}$ NMR $\left(\mathrm{CDCl}_{3}, 400 \mathrm{MHz}\right)$

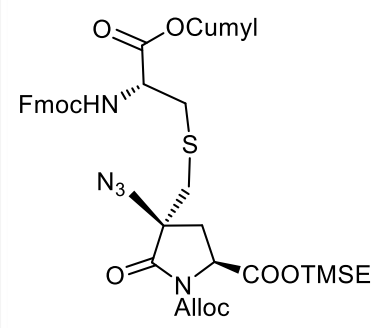

$3 \mathbf{b}$

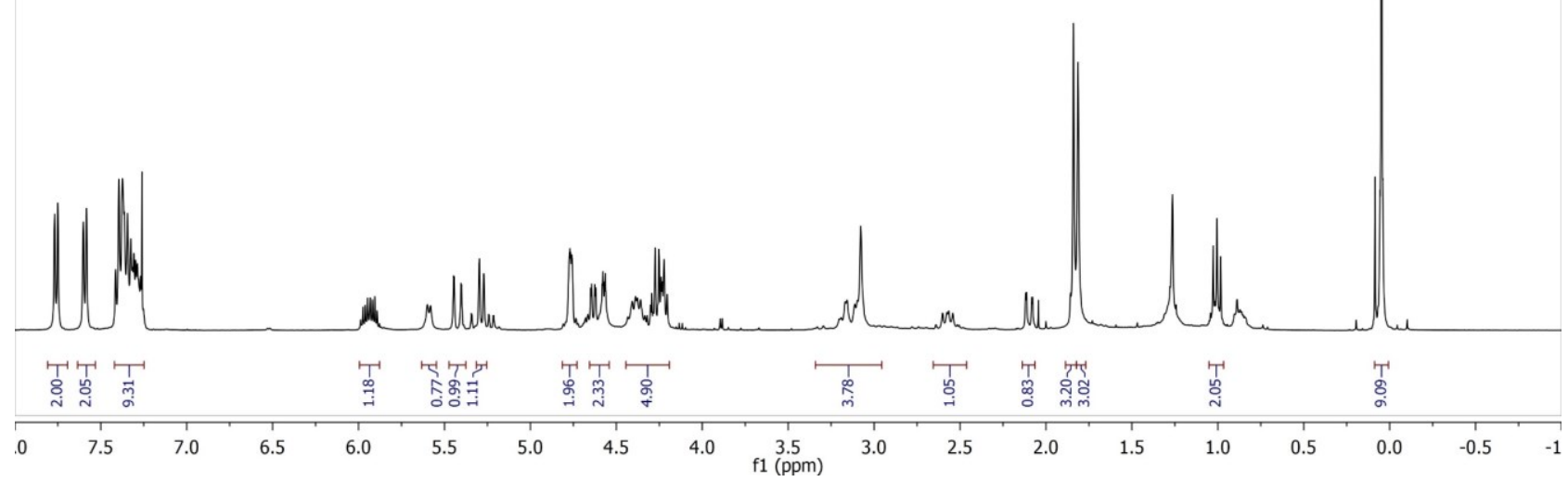

${ }^{13} \mathrm{C}\{1 \mathrm{H}\}$ NMR $\left(\mathrm{CDCl}_{3}, 101 \mathrm{MHz}\right)$

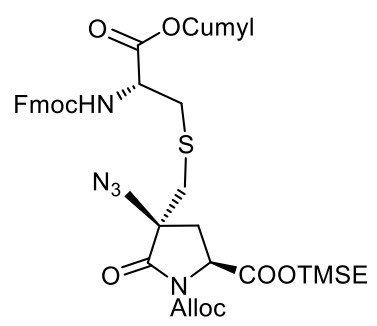

3b

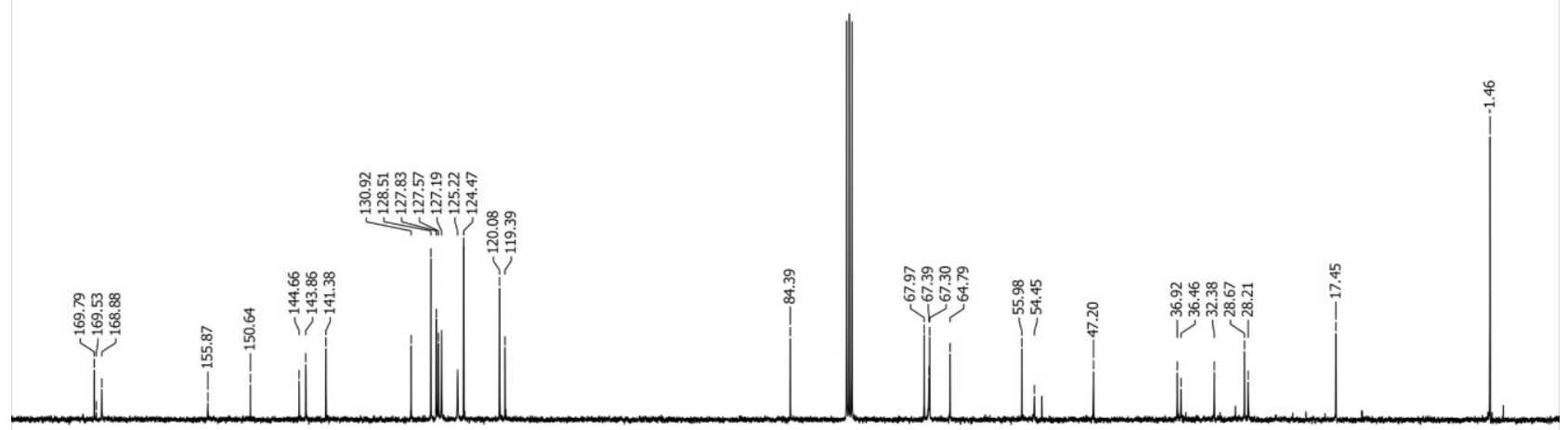

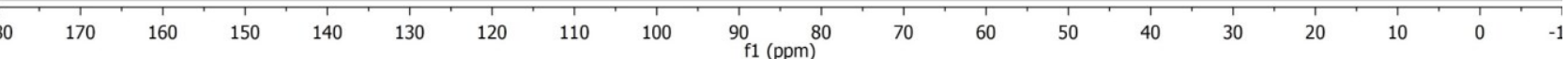


${ }^{1} \mathrm{H}$ NMR (MeOD, $400 \mathrm{MHz}$ )

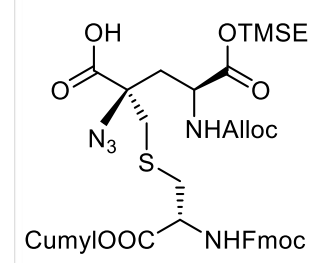

$15 a$

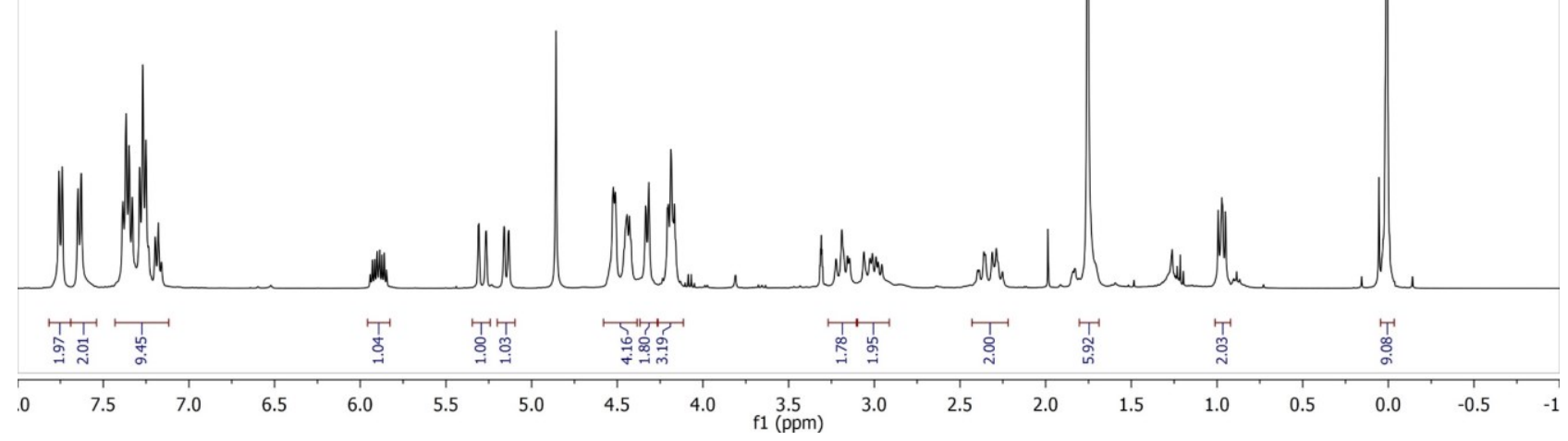

${ }^{13} \mathrm{C}\{1 \mathrm{H}\}$ NMR (MeOD, $\left.101 \mathrm{MHz}\right)$

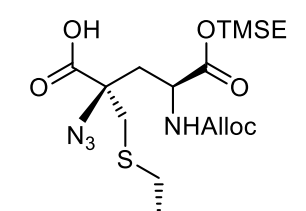

CumylOOC $\overbrace{\text { NHFmoc }}$

$15 a$

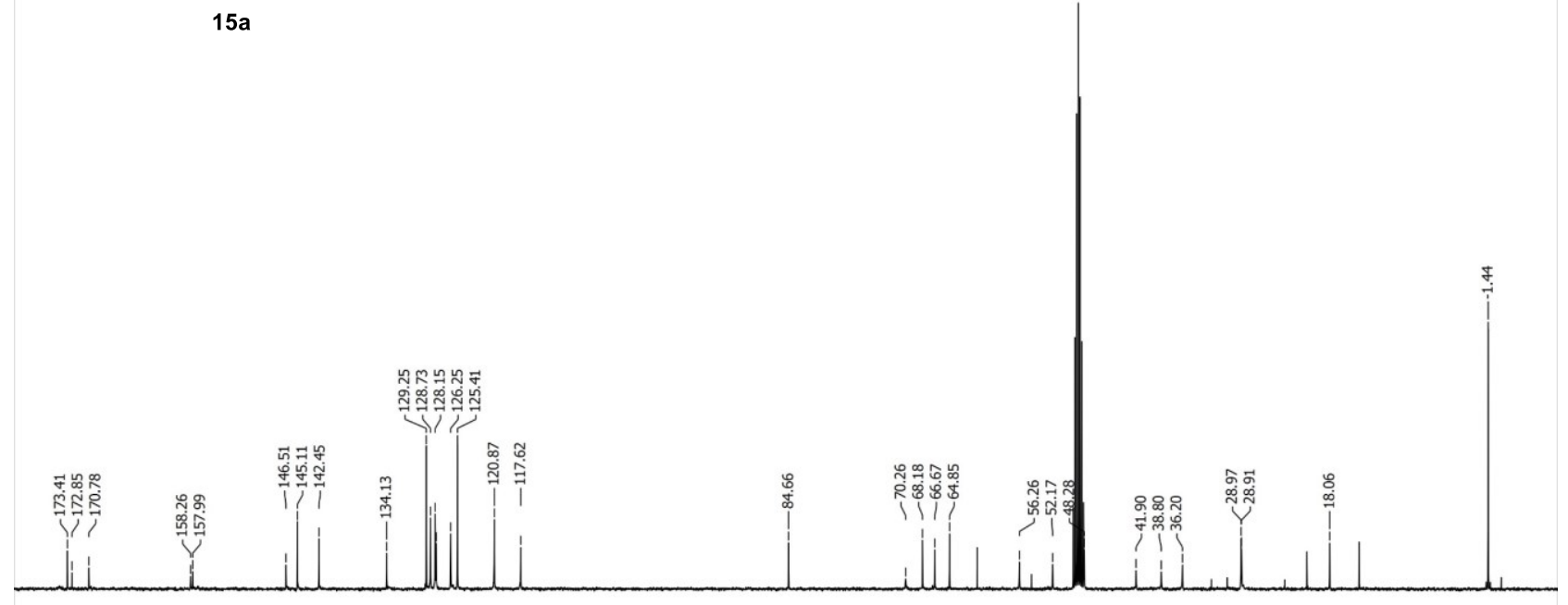

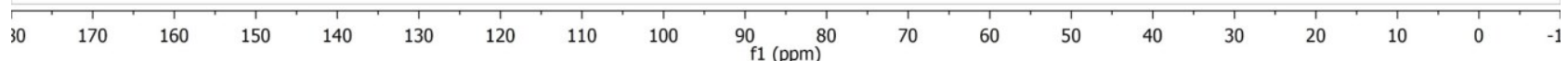




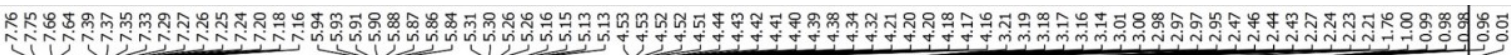

${ }^{1} \mathrm{H}$ NMR (MeOD, $\left.400 \mathrm{MHz}\right)$

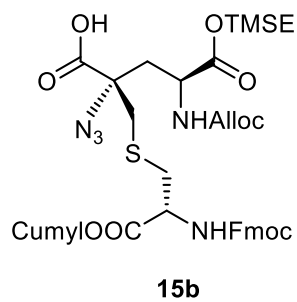

$15 b$

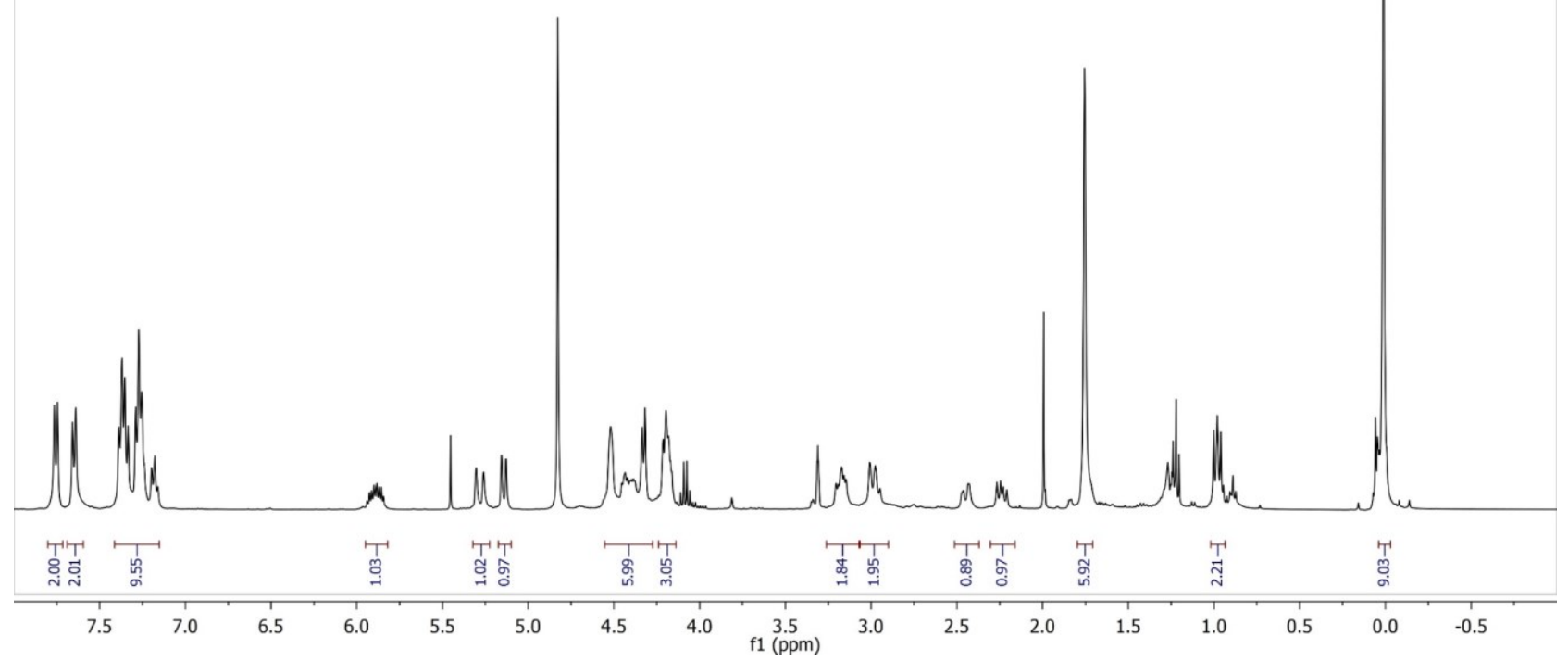

${ }^{13} \mathrm{C}\{1 \mathrm{H}\}$ NMR (MeOD, $\left.101 \mathrm{MHz}\right)$

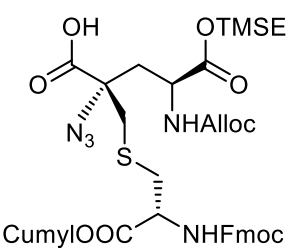

15b
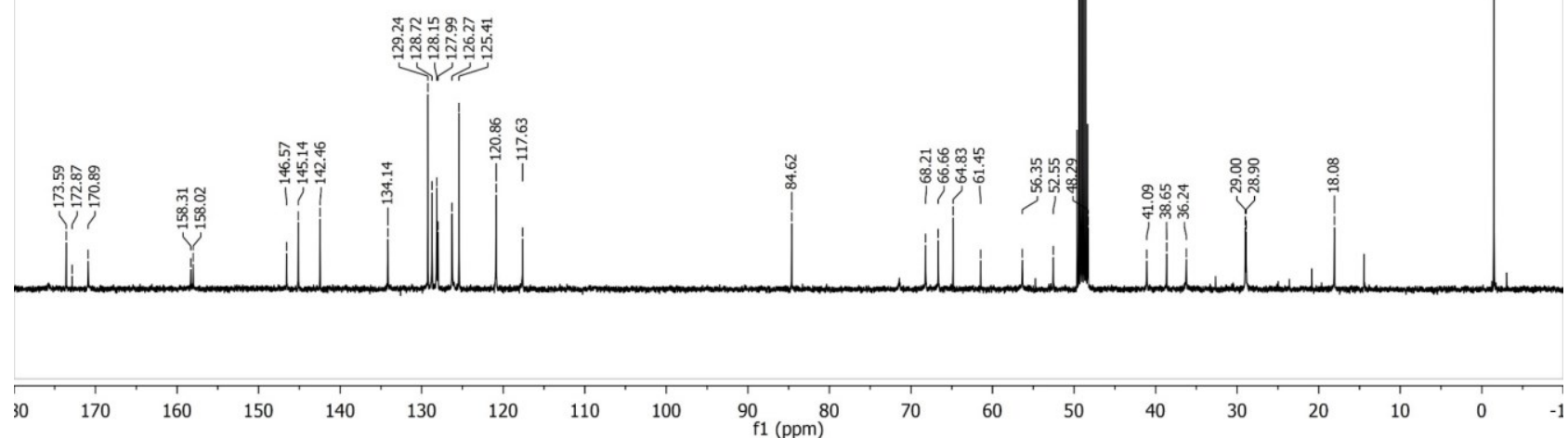


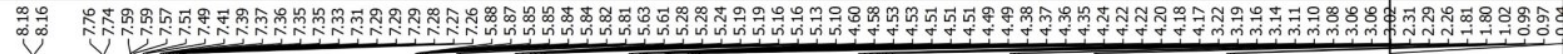

${ }^{1} \mathrm{H} \mathrm{NMR}\left(\mathrm{CDCl}_{3}, 400 \mathrm{MHz}\right)$

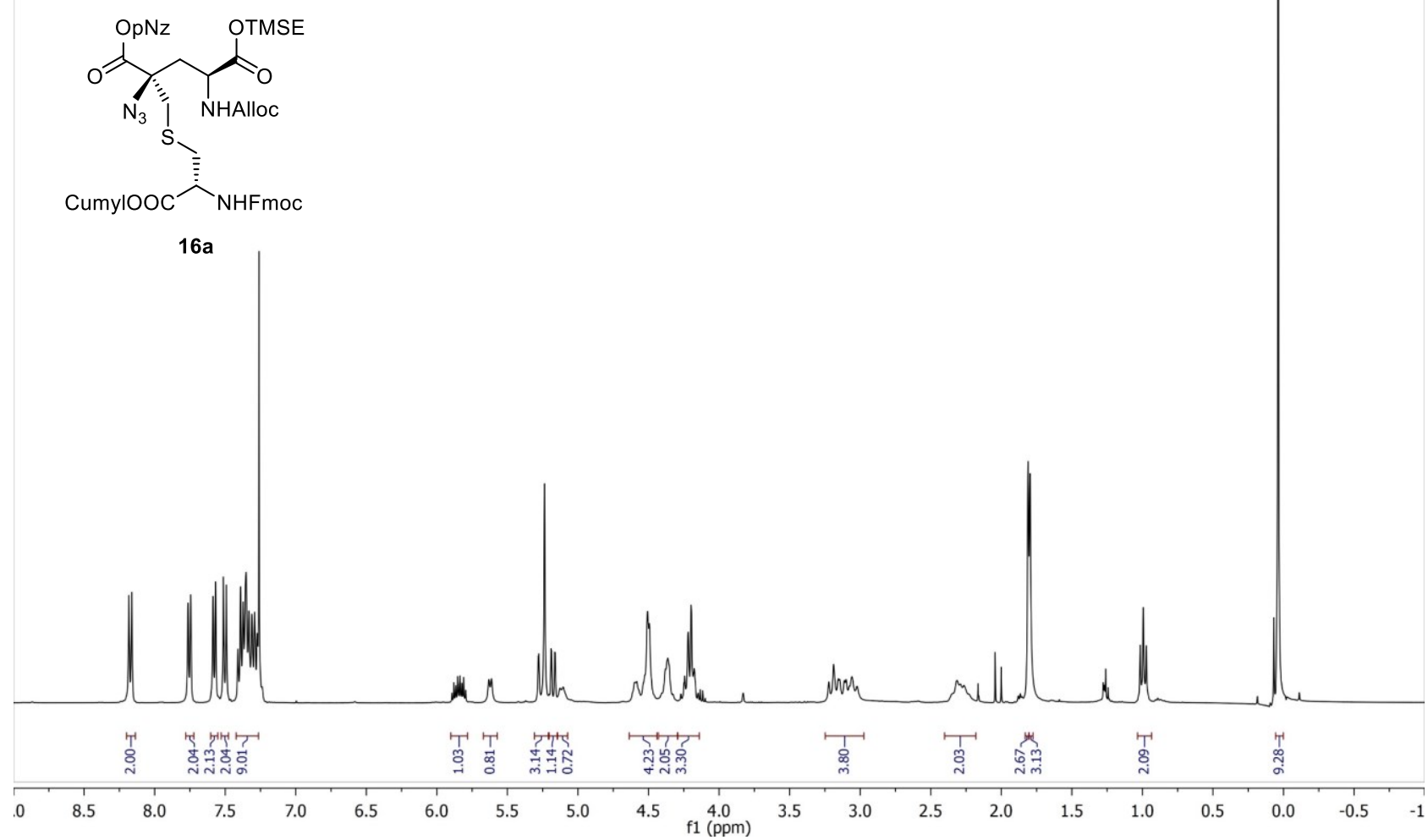

${ }^{13} \mathrm{C}\{1 \mathrm{H}\} \mathrm{NMR}\left(\mathrm{CDCl}_{3}, 101 \mathrm{MHz}\right)$

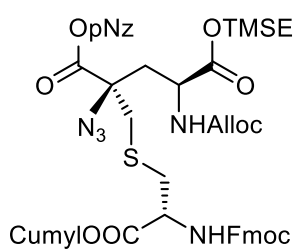

$16 a$
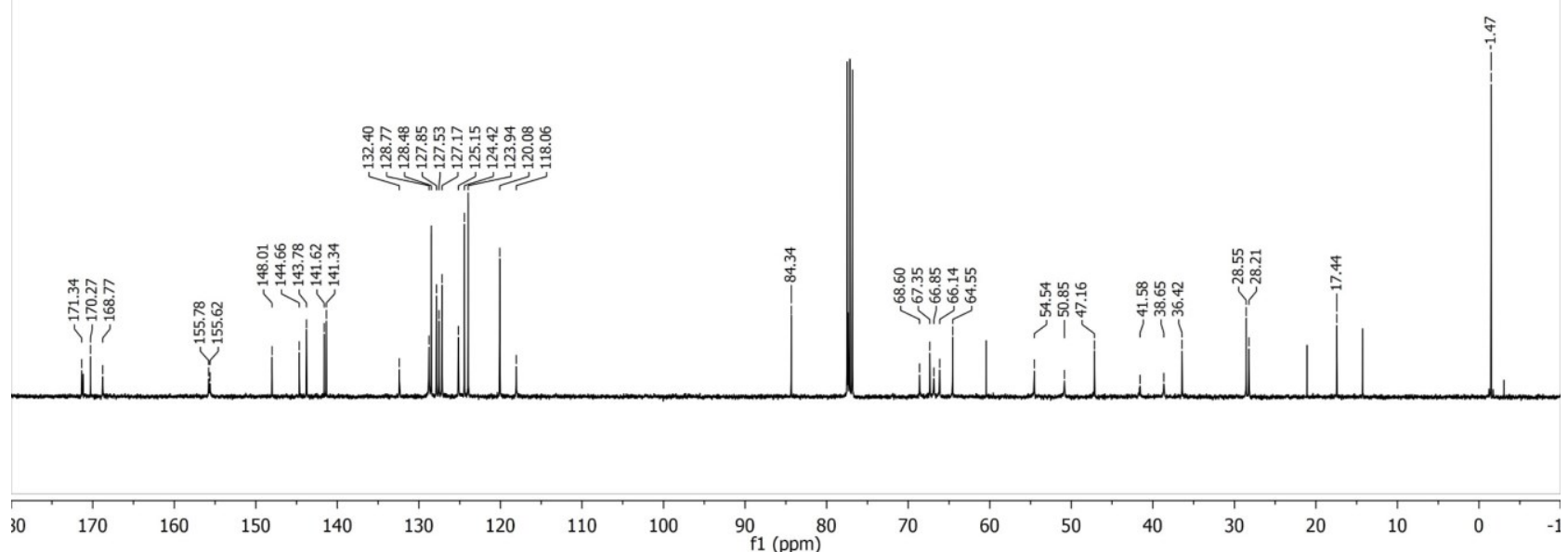
${ }^{1} \mathrm{H}$ NMR $\left(\mathrm{CDCl}_{3}, 400 \mathrm{MHz}\right)$
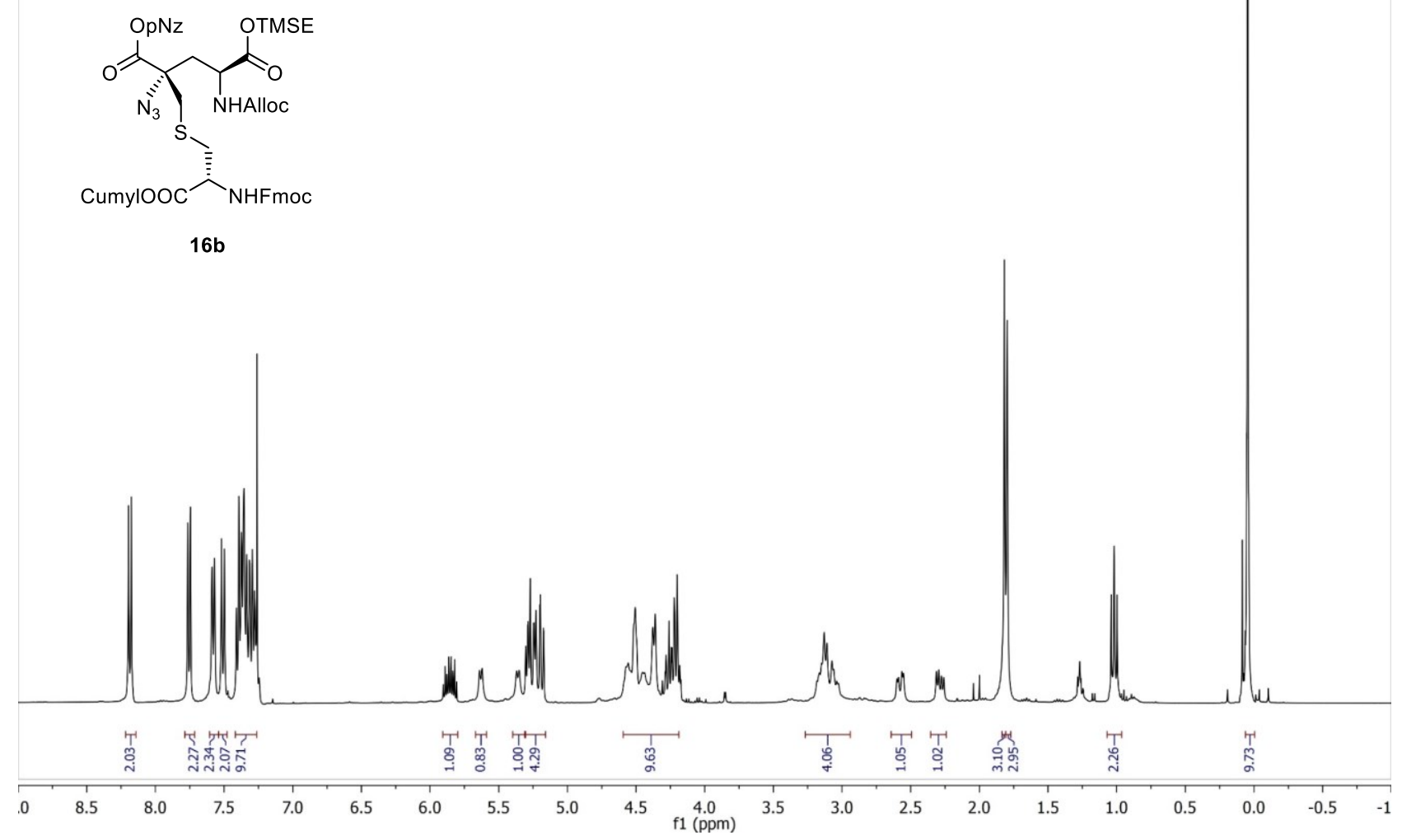

${ }^{13} \mathrm{C}\{1 \mathrm{H}\} \mathrm{NMR}\left(\mathrm{CDCl}_{3}, 101 \mathrm{MHz}\right)$
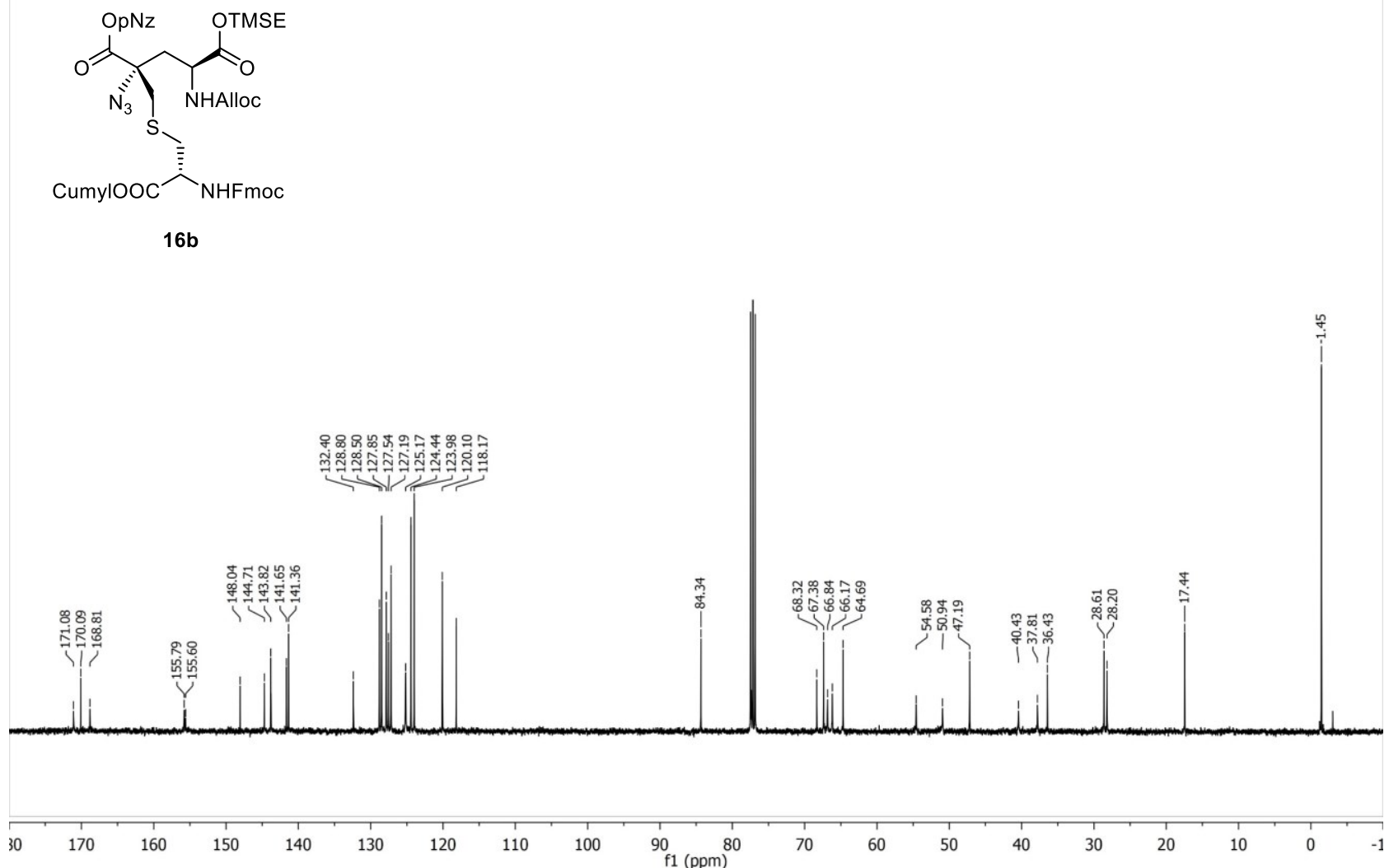
${ }^{1} \mathrm{H}$ NMR (MeOD, $\left.400 \mathrm{MHz}\right)$

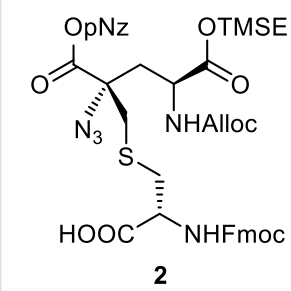

${ }^{3} \mathrm{C}\{1 \mathrm{H}\}$ NMR (MeOD, $101 \mathrm{MHz}$ )
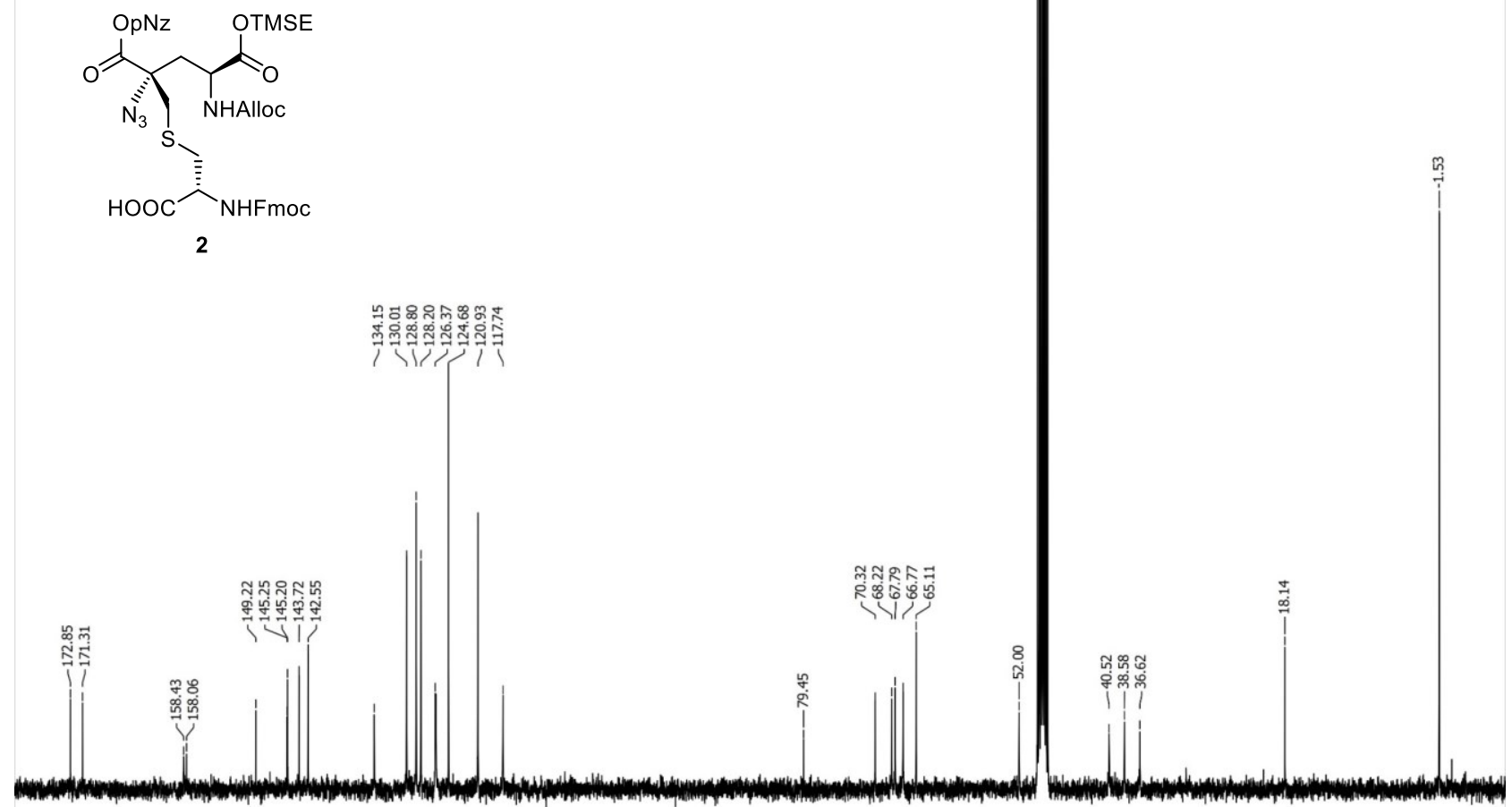

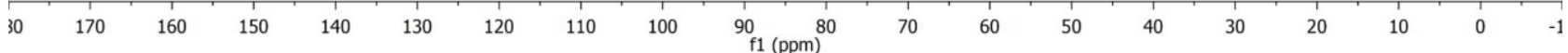




\section{${ }^{1} \mathrm{H}$ NMR (MeOD, $400 \mathrm{MHz}$ )}

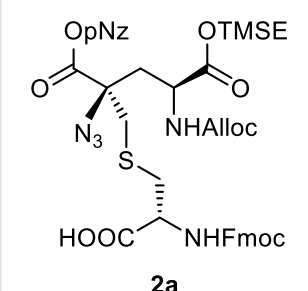

2a

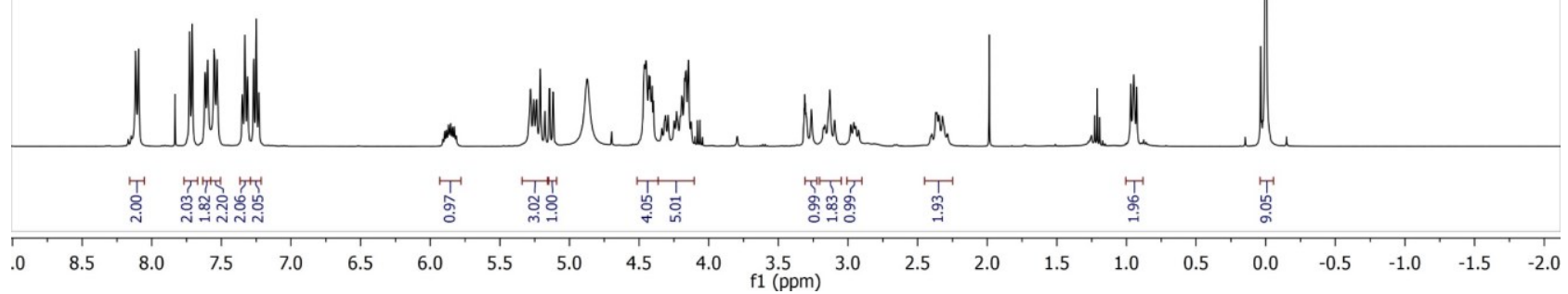

\section{${ }^{13} \mathrm{C}\{1 \mathrm{H}\}$ NMR (MeOD, $101 \mathrm{MHz}$ )}
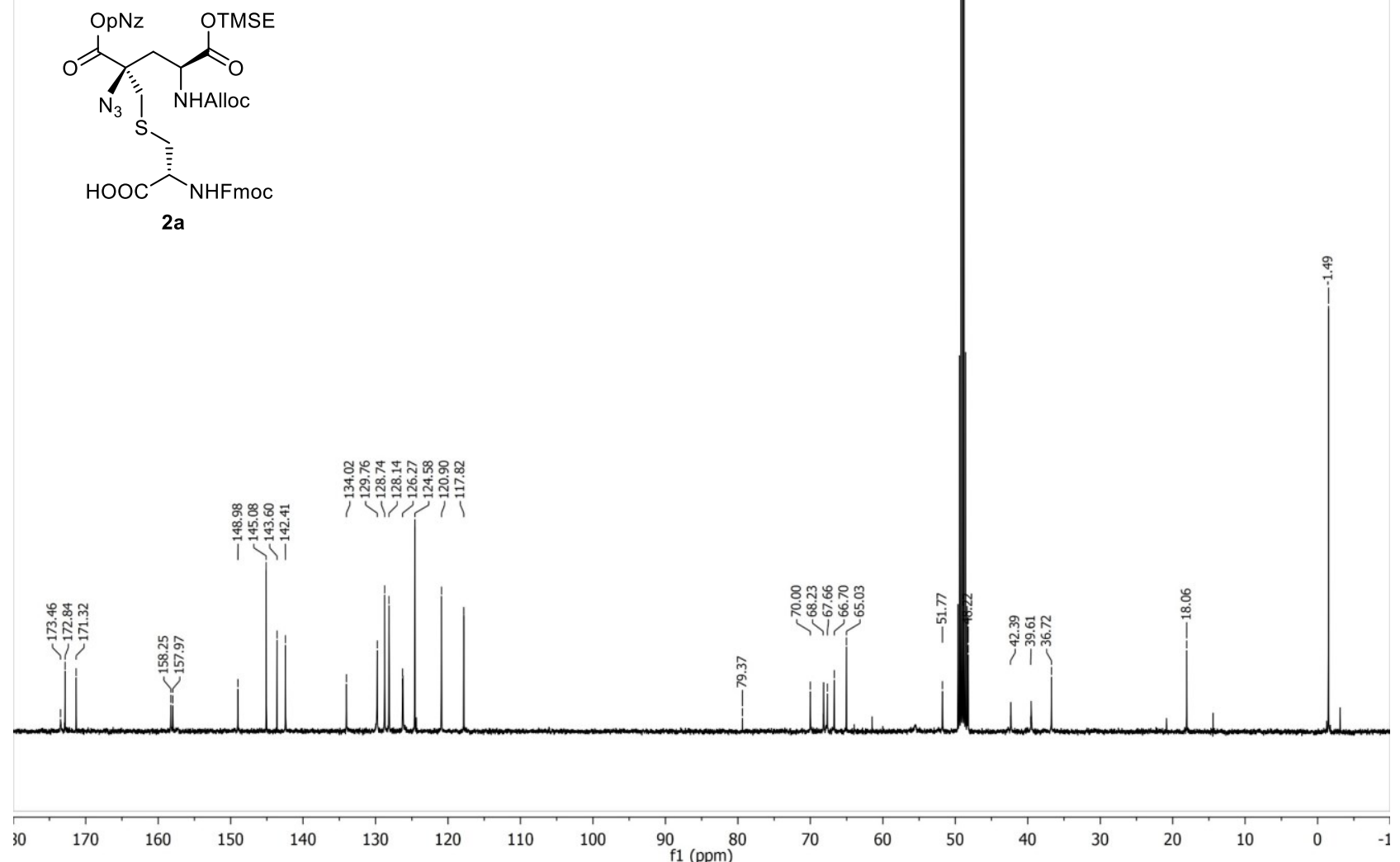
Mass Spectra of compounds
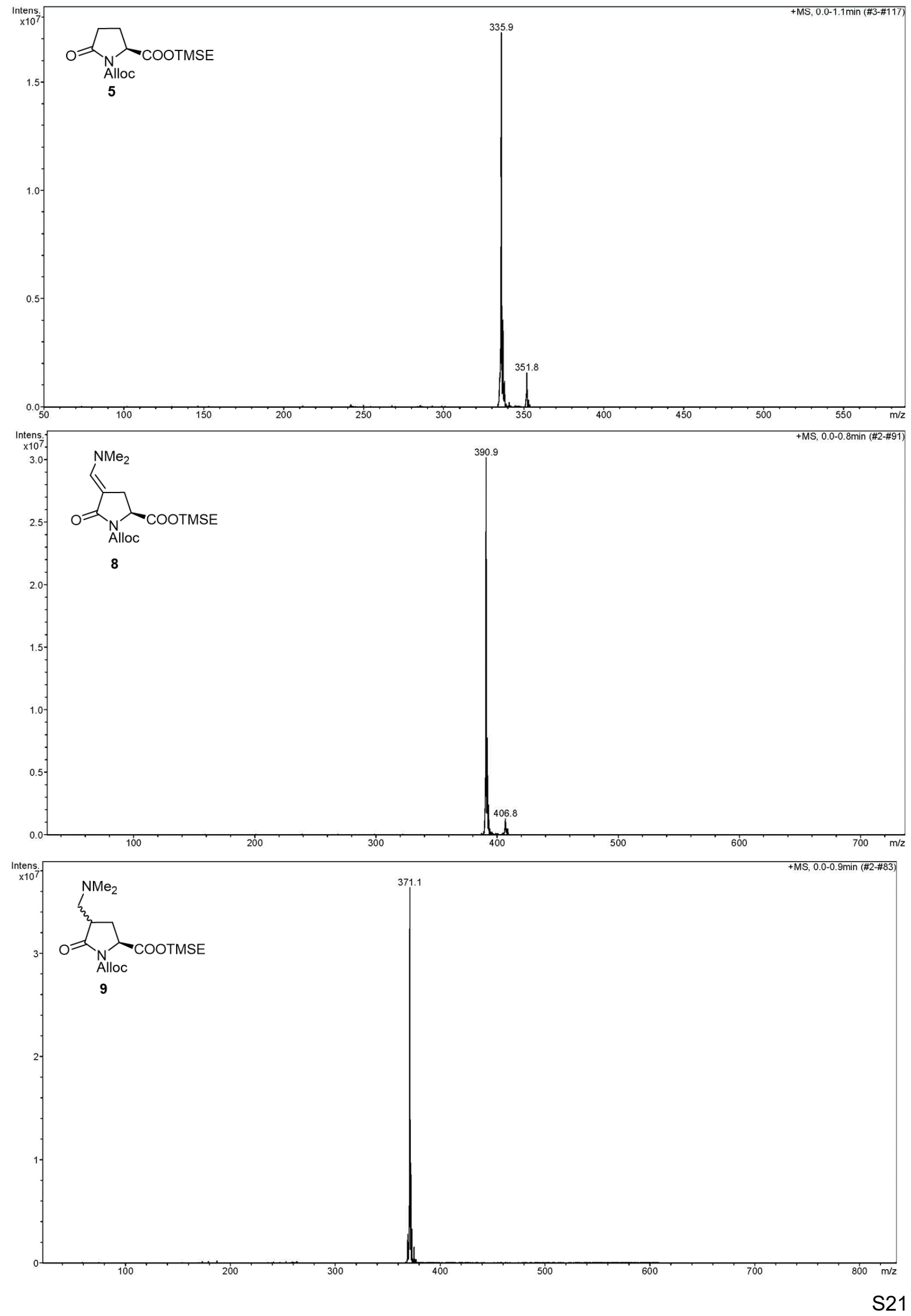

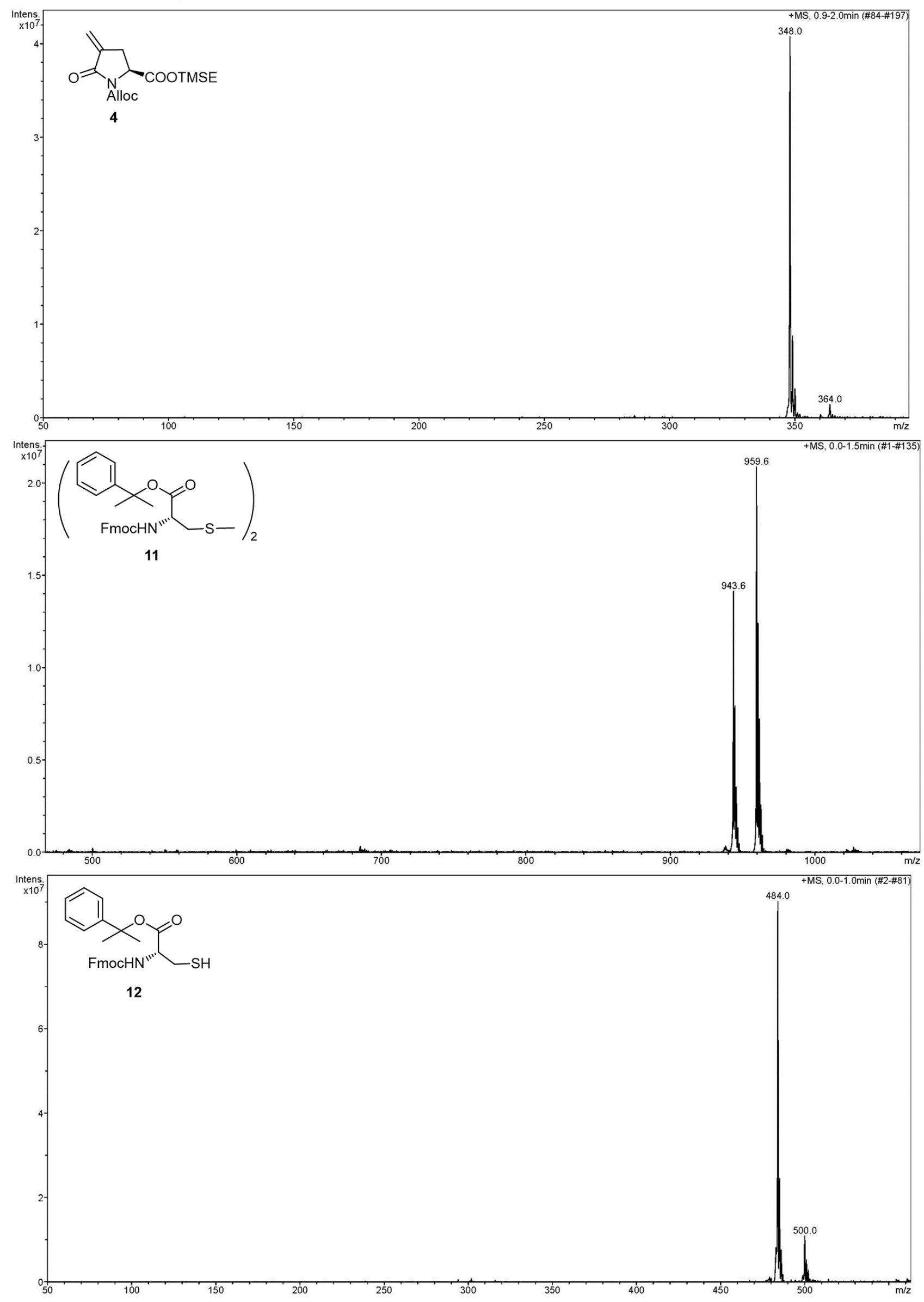

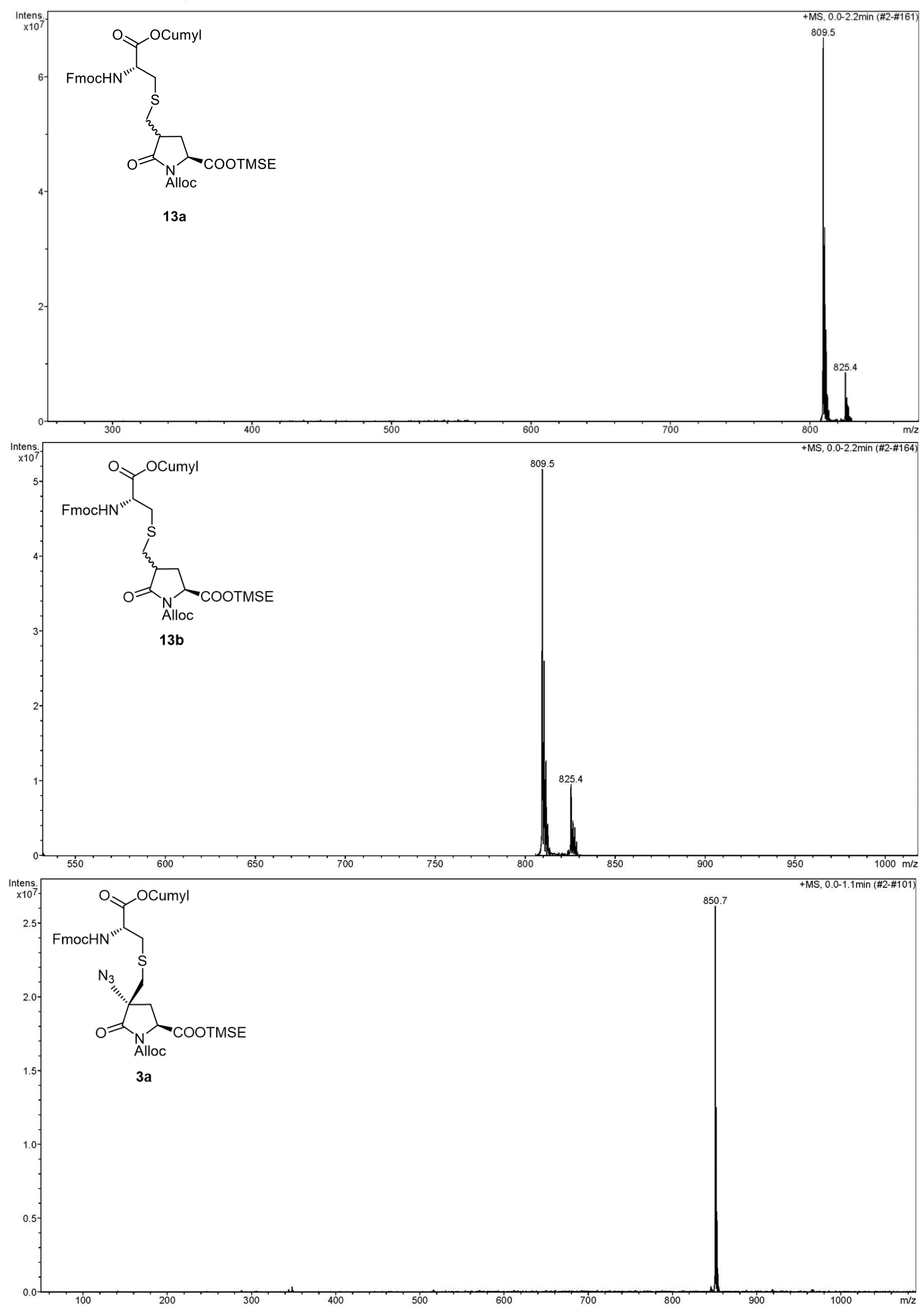

$\mathrm{S} 23$ 

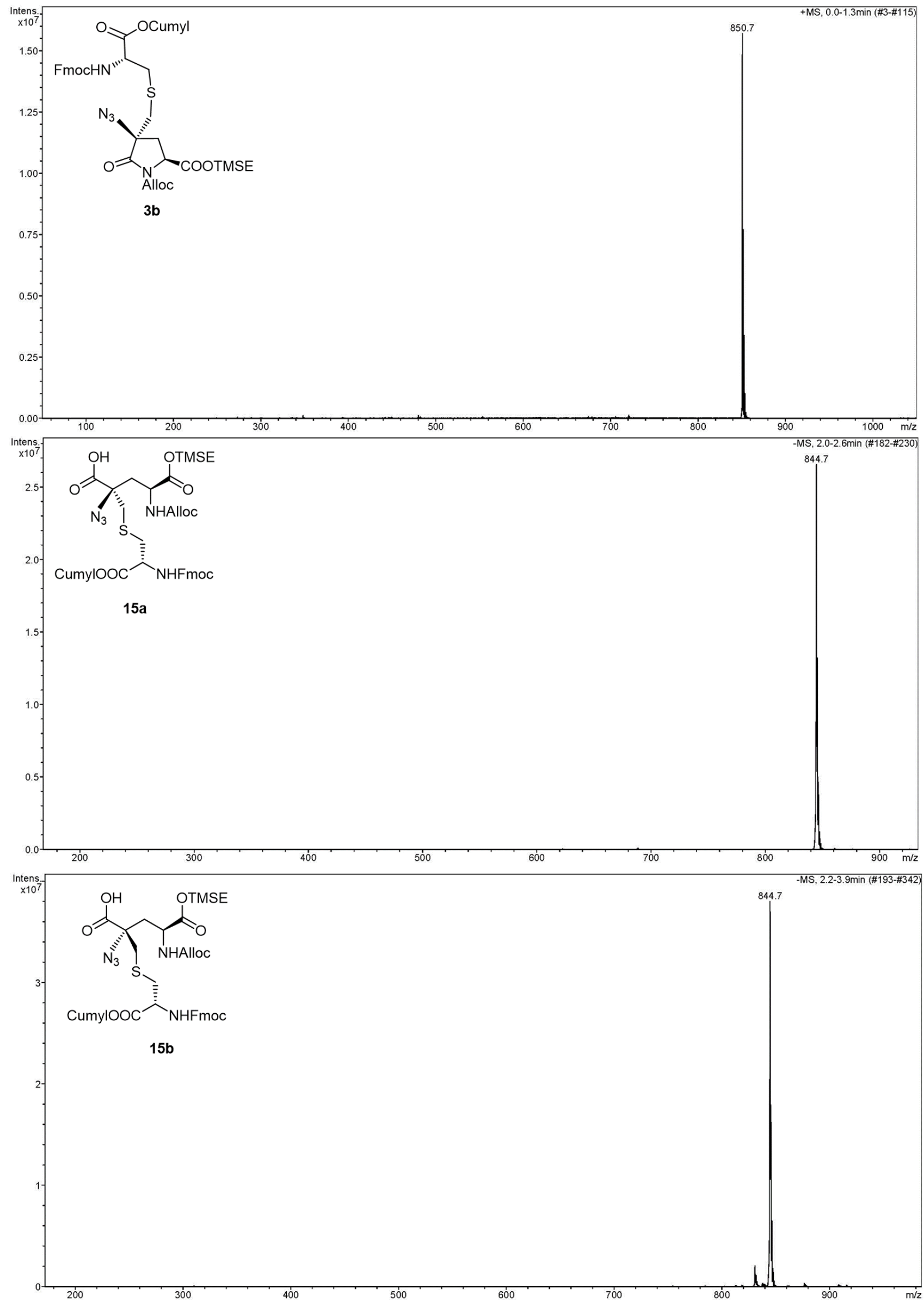


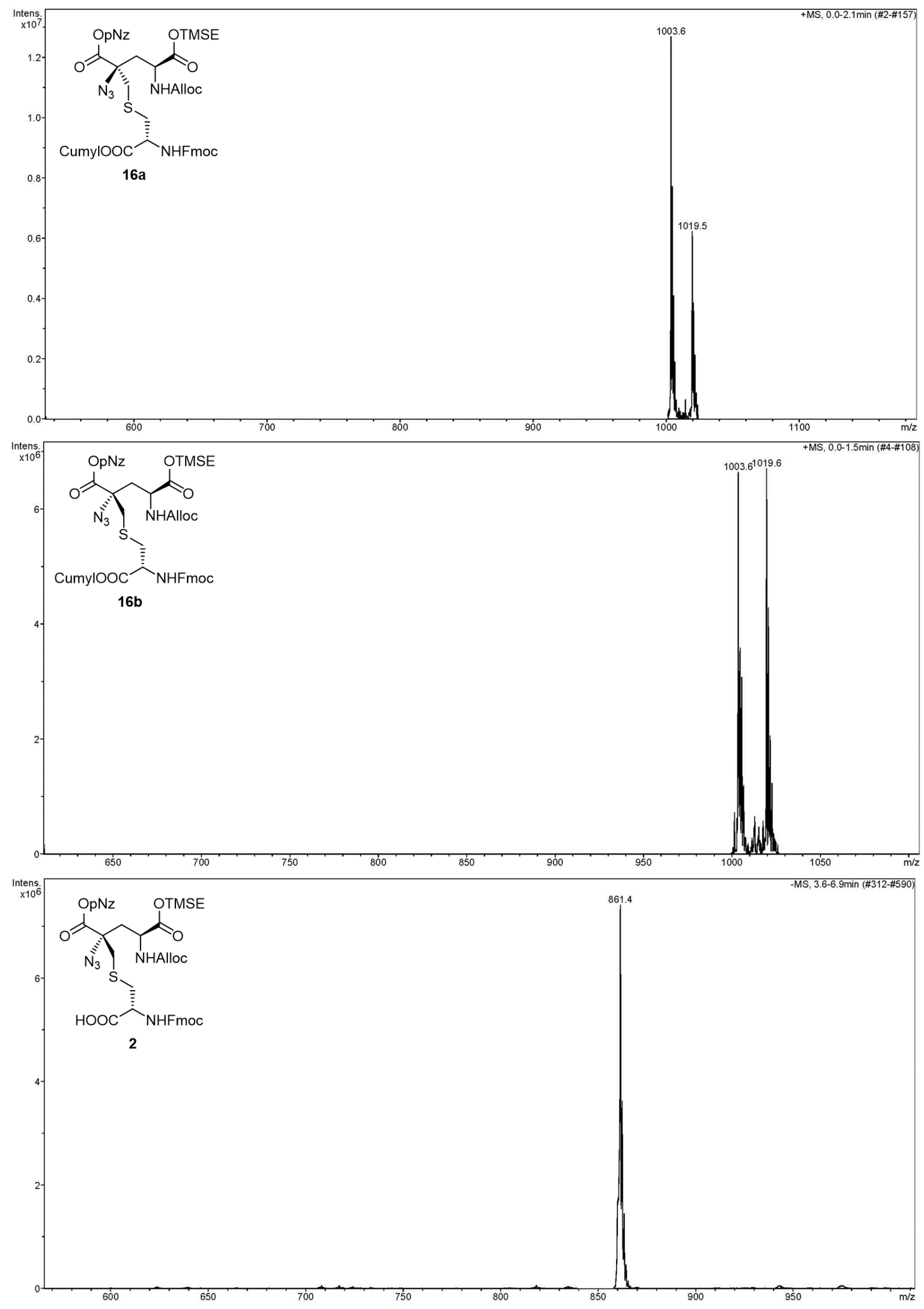




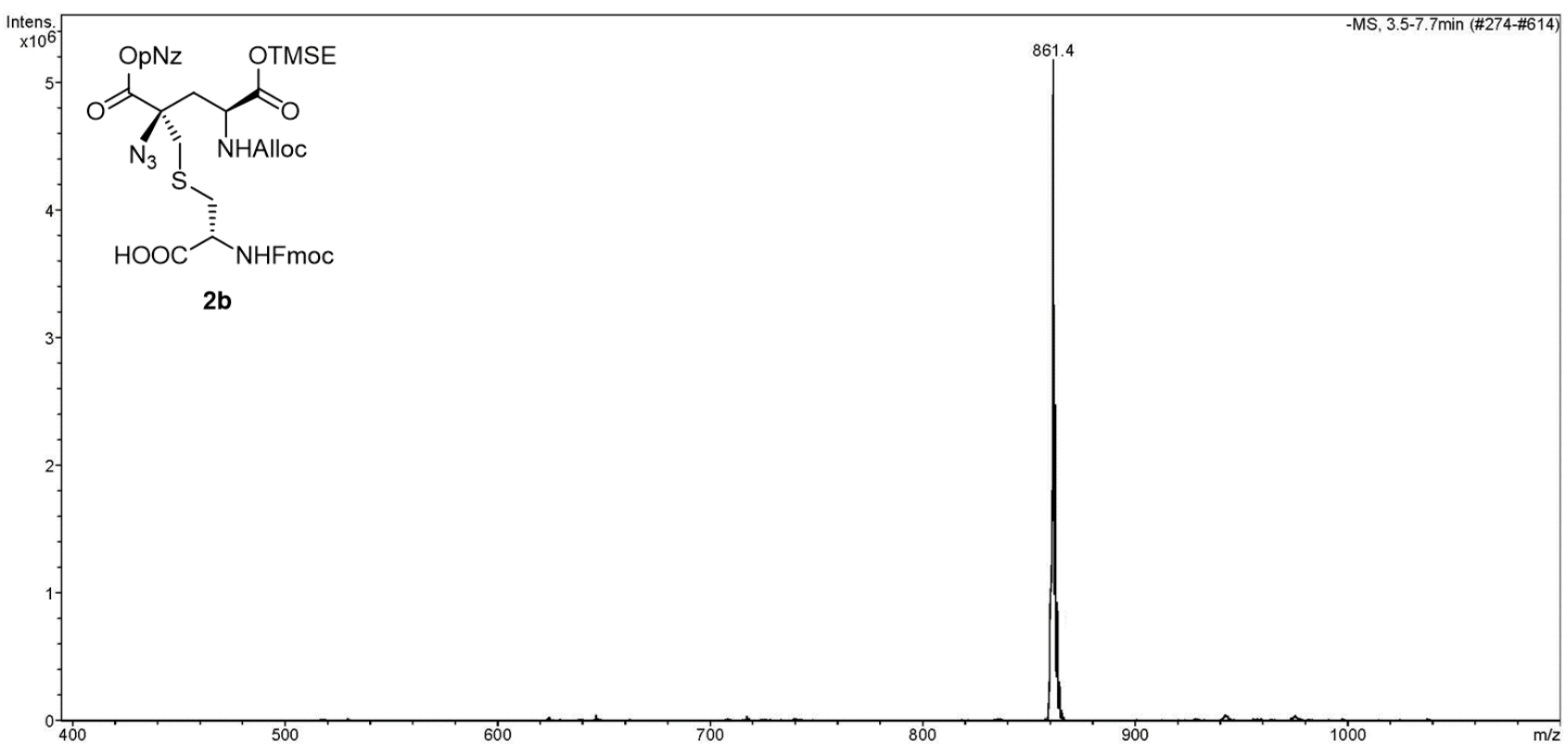

S26 